Performance evaluation of surgical techniques for treatment of scapholunate instability in a type II wrist.

R. Leonardo-Diaz ${ }^{1}$, M.T. Alonso-Rasgado ${ }^{2}$, D. Jimenez-Cruz ${ }^{1}$, C.G. Bailey ${ }^{2}$, S. Talwalkar ${ }^{3}$

${ }^{1}$ School of Materials, The University of Manchester, Manchester, United Kingdom.

${ }^{2}$ School of Engineering and Materials Science, Queen Mary University of London, London, United Kingdom

${ }^{3}$ Wrightington Hospital, Wrightington Wigan and Leigh NHS Foundation Trust, Lancashire, United Kingdom

Corresponding Author:

Professor M. T. Alonso Rasgado

Professor of Mechanical Engineering

School of Engineering and Materials Science

Queen Mary University of London

London E1 4NS

UK

email: t.alonso@qmul.ac.uk 


\section{Performance evaluation of surgical techniques for treatment of scapholunate instability in a type II wrist}

\section{INTRODUCTION}

Scapholunate (SL) dissociation occurs when the scaphoid and lunate move excessively in relation to one another; it is caused by the injuring of the scapholunate interosseous ligament (SLIL) and affects carpal instability [1]. Often, SLIL injuries are not diagnosed or treated during an acute phase of injury because it can take three to twelve months after trauma before dynamic instability develops and the SL dissociation can be detected radiologically; additionally, in some cases people may have a tear of the SLIL but not present clinical symptoms [2]. Scapholunate instability has been reported to occur in approximately $5 \%$ of cases of wrist sprains [2] and is believed to be as common as scaphoid fractures [3]. Some authors have theorised that the effect on carpal instability after SLIL tear may be moderated by the bone geometry, which may help to explain why some patients go on to progressive instability whilst others do not [4-6]. In a cadaver study, Werner et al (2007) determined that bone geometry at the distal radius and proximal scaphoid may have a moderate effect on the carpal instability after sectioning [4]. Rhee et al (2009) found from a clinical review that patients possessing an additional articulation between the lunate and hamate have a lower incidence of DISI following a complete SLIL tear [5]. Other researchers, however, have found no link between bone geometry and carpal instability following SL injury [7].

The wrist has been categorised into type I and type II depending on the morphology of the lunate bone. A type I lunate is identified as having a shortest distance between the capitate and triquetrum (C-T distance) on a posterior anterior radiograph of $\leq 2 \mathrm{~mm}[8,9]$, Figure 1-a. A type II lunate has an extra facet that articulates with the hamate [8-11], and a C-T distance $\geq 4 \mathrm{~mm}$ and $\leq 8.9 \mathrm{~mm}[8,9]$, Figure 1-b. A third, intermediate group, laying between Type I and Type II wrists has also been identified by some researchers [7]. There is a clear difference between a type I and II wrist in articular cartilage contact Figure 1-c and Figure 1d, $[9,10]$. No registry of the incidence of type I and type II wrists has been set up, but some previous studies on lunate morphology reported type II wrists to be more common, with the proportion of the frequency of a type II lunate being $63 \%$ to $73 \%$ [11-14]. The categorisation is seldom mentioned in the literature and only recently have some authors discussed the incidence, variation, associated pathologies and the clinical importance of the extra facet, 
including some studies that relate lunate morphology to carpal mechanics. Statistically, there is no correlation between lunate type and gender, age, hand dominance or occupation $[8,15]$.

Classically, carpal mechanics may be described using two main motion theories, row theory and column theory, with type I wrists considered to exhibit row mechanics and type II column mechanics [8]. In row theory, the carpal bones are divided into two rows of adjacent bones. The proximal row consists of the scaphoid, the lunate, the triquetrum and the pisiform; the distal row comprises of the hamate, capitate, trapezium and trapezoid, see Figure 2-a [8, 15]. During clench fist ulnar deviation (volar view), the proximal row rotates and slides over the concave surface of the radius, meanwhile the distal row rotates in the same direction sliding over the proximal row allowing the motion Figure 2-b. In column theory, the carpal bones are distributed into three columns: the lateral column consists of the scaphoid, the central column consist of lunate, capitate, hamate, trapezium and trapezoid, and the medial column consists of the triquetrum and pisiform Figure 2-c. Column theory suggests that during ulnar deviation (volar view), the central column rotates clockwise (in the ulnar direction); simultaneously the medial column slides over the hamate to the dorsal side making space for the moving bones, meanwhile the scaphoid tilts towards the vertical by the pulling of the ligament that joins it with trapezium and trapezoid, [8, 15] Figure 2-d. To date, validation of these theories has only been performed using plane X-ray and fluoroscopic studies $[8,11,15]$.

A number of surgical techniques have been employed to reconstruct the scapholunate interosseous ligament following injury in cases where the SLIL is nonrepairable but the SL dissociation is reducible, these include the Brunelli tenodesis method and the modified Brunelli technique (MBT) [16-18] and more recently the Corella [19] and the SL axis methods (SLAM) [20]. Existing in vitro studies comparing the performance of the techniques have failed to report whether the specimens considered were type I or type II wrists [20].

The aim of the current study is to investigate the performance of three tenodesis techniques, modified Brunelli, Corella and scapholunate axis (SLAM) methods used to repair SL ligament disruption for type I and II wrists. The ability of the techniques to restore wrist stability (SL gap and angle) following SLIL tear is assessed using finite element (FE) models developed to simulate the reconstruction techniques in addition to SL tear and intact wrist scenarios. The techniques are assessed for both type I and II wrists based on FE model predictions of SL gap and angle at both dorsal and volar sides and scaphoid-lunate contact 
area. In addition, the carpal mechanics of the wrists is investigated using the FE models in order to determine whether application of the ligament reconstruction techniques alters wrist kinematics. Moreover, the current study aims to determine if it is appropriate to employ the same technique for both type I and II wrists or if the choice of ligament reconstruction technique should potentially be made based on wrist type.

\section{MATERIALS AND METHODS}

Previously, the authors developed a finite element model, validated with data from a cadaveric study, which was used to investigate the performance of three surgical SLIL reconstruction techniques used to treat scapholunate instability in type I wrists [21]. The current study is an extension of this investigation but for type II wrists.

Six finite element models were developed to simulate the intact SL ligament type II wrist in neutral posture and five ulnar deviated clenched fist position cases: intact SL ligament, SLIL sectioning, modified Brunelli, Corella and scapholunate axis (SLAM) tenodesis techniques.

Dorsal and volar SL gap and angle were obtained for the six scenarios from the type II wrist models enabling the performance of the reconstruction techniques to be assessed and compared with the results from models of type I wrists, obtained previously by the research group [21]. In addition, contact areas of lunate and scaphoid bones were obtained from the type I and II models enabling the difference between type I and type II wrists to be elucidated and performance of the reconstruction techniques to be further assessed. In addition, the carpal mechanics is investigated in the context of row/column theory for type I and type II wrist in order to study the kinematics of the 2 types of wrist before and following the application of the virtual reconstruction techniques.

\section{Finite Element Modelling}

The geometry for the 3D FE models was constructed from Computed Topography (CT) scan images of a type II wrist from the left hand of a subject with no history of injury. The classification of a type wrist II for the subject was based on the criteria described by Galley et al. [8], that being that the minimal distance between capitate-triquetrum (C-T distance) of the subject be $\geq 4 \mathrm{~mm}(6.5 \mathrm{~mm})$, Figure 3 -a. The CT images comprised 232 slices with a 
thickness of $1 \mathrm{~mm}$ and transverse resolution of $512 \times 512$, including the distal end of radius and ulna to the metacarpals.

CT scan images were imported into 3-D image segmentation and processing software. Semiautomatic segmentation was performed using a thresholding technique to construct the geometries with images resampled with a pixel spacing of $0.4 \mathrm{~mm} \times 0.4 \mathrm{~mm} \times 0.4 \mathrm{~mm}$ in order to optimise element size and mesh quality. Two masks were created to produce 3D surface representations of the cortical and cancellous bone for each of the 15 bones, radius, ulna, the eight carpals and the 5 metacarpals bones. Figure 3-b. The surface representations were imported into Abaqus 6.14 (Dassault Systemes, Rhode Island) and then converted into a solid mesh. Parts representing the bones were assembled in Abaqus to create the wrist model; each part was meshed using solid tetrahedral elements (C3D4) Figure 3-c.

Cartilage layers were modelled with wedge elements (C3D6) by offsetting the articulating surfaces on the external layer of the cortical bone by half the minimum gap between the articulating bones [22], Figure 3-d, to create a solid layer. This solid layer was meshed with wedge elements (C3D6). Surface-to-surface contact was defined between the cartilage surfaces on each articulation for the proximal carpal bones to allow free movement of scaphoid and lunate. A friction coefficient of 0.002 was considered for all the cartilage surface-to surface interactions [23]. Distal carpal bones were considered tied to simulate the minimal motion between each pair of bones, as reported in previous studies [24].

Thirty-one sets of ligaments were modelled with two-node springs elements, including 10 extrinsic, 16 intrinsic, and 5 interosseous ligaments. The origin and insertion points were estimated based on anatomical studies $[25,26]$. The anatomic relationship of the volar triquetrocapitate $(\mathrm{T}-\mathrm{C})$ and the volar triquetrohamate $(\mathrm{T}-\mathrm{H})$ was set in accordance with the taxonomy reported by Nakamura [27]. Each set of ligaments was represented by multiple elements to distribute the force over an area to avoid stress concentrations, Figure 3-e.

Three ligaments, volar radiocaphocapitate, dorsal radiocarpal and intercarpal, were modelled using shell elements to simulate the wrapping of these ligaments around scaphoid, lunate and hamate, capitate and scaphoid respectively. All 31 sets of ligaments were defined as operating in tension only, with stiffness from 10 to $325 \mathrm{~N} / \mathrm{mm}$ in accordance with the literature [28-30], (supplementary Table, Ligaments included in the model). For the three ligaments meshed with shell elements, the stress-strain relationships were calculated from their stiffness and the corresponding cross sectional areas [26]. Contact was defined between 
the inner surface of the 2D ligaments and the outer surfaces of the bones as a frictionless surface-to-surface interaction.

Linear and elastic isotropic behaviour was assumed for the bone and cartilage; a Young's modulus of $18 \mathrm{GPa}$ was employed for cortical bone and $100 \mathrm{MPa}$ for the cancellous bone, a Poisson's ratio of 0.2 and 0.25 , was used respectively [23, 29, 31]. Cartilage was defined as a hyper-elastic material, for which Mooney-Rivlin parameters C10 and C01 were assigned of 4.1MPa and 0.41MPa respectively [23, 29].

\section{Loading, Boundary Conditions and Mesh Sensitivity Analysis}

The proximal ends of radius and ulna were fully constrained. Loads to simulate clenched fist posture have been proposed in previous studies [21] and these were used in this study. The forces applied to the numerical model were determined based on those employed in a previous cadaveric study to produce the clenched fist posture [21]. In the cadaveric study, 6 tendons/tendon groups were exposed to facilitate loading to produce the clenched fist posture. Loading weights of $15 \mathrm{~N}$ were attached separately to flexor digitorum superficialis (FDS), flexor digitorum profundus/flexor pollicis longus tendon (FDP/FPL), flexor carpi ulnaris (FCU) and extensor carpi ulnaris (ECU) while a load of $20 \mathrm{~N}$ was applied to the extensor digitorum communis (EDC). To simulate this loading condition in the numerical model, loads were applied to the proximal region of pisiform and to the dorsal base of the fifth metacarpal; the remaining three loads used to produce the clenched fist posture were applied by equally distributing the forces on the proximal end of the corresponding metacarpal bones. A force of $3 \mathrm{~N}$ was applied on the proximal end of metacarpal 1 and, similarly, forces of magnitude $11.75 \mathrm{~N}$ on metacarpals 2 to 5 . Additionally, vertical loads of $15 \mathrm{~N}$ were added; one load applied at the proximal region of pisiform and other to the dorsal base of the fifth metacarpal, Figure 3-e. A mesh sensitivity analysis was performed using the intact model in neutral position to ensure the accuracy of the predictions. Lunate bone contact area was considered and mesh density was increased until the value of the contact area changed by less 5\% (Figure 4). This mesh density was then employed for the subsequent analysis. The final model consisted of a total of 507,171 elements (10,372 wedge elements of type C3D6 for the cartilage, 496,491 tetrahedral elements of type C3D4 for the solid bones, 301 quadrilateral elements of type S4R and 7 triangular elements of type S3 for the ligaments represented by shell elements) and 111,404 nodes. The lunate - scaphoid cartilage surface was modelled using 164 C3D6 wedge elements (6-noded linear triangular prism), representing a total area 
of $57.26 \mathrm{~mm}^{2}$. The average volume of the elements on the lunate - scaphoid surface was $0.22 \mathrm{~mm}^{3}$ and the average area of the element face (on the cartilage surface) was $0.2 \mathrm{~mm}^{2}$.

\section{Virtual Reconstruction}

Six cases were modelled: Intact-neutral clenched fist posture (Figure 5-a), intact-ulna deviate clenched fist position (Figure 5-b), SLIL sectioning-ulna deviate clenched fist position (Figure 5-c) and three reconstruction techniques (Corella, modified Brunelli and SLAM). For the SLIL sectioning case, the SLIL was fully removed so there was no association between the scaphoid and lunate via ligament, Figure 5-c. The virtual reconstruction techniques were modelled as described in previous studies [21]. For the virtual MBT technique, a 3-mmdiameter hole is drilled from the palmar tuberosity to the dorsal point of insertion of dorsal SLIL in the scaphoid. The graft was positioned, passing from volar to dorsal side of the scaphoid through the hole made, then connected to the lunate bone at the dorsal side, Figure 5-d. The tendon graft was simulated using solid cylindrical elements.

In the Corella technique, two holes in the scaphoid and lunate bones are drilled. The tendon graft is then passed sequentially through the scaphoid and the lunate connecting both bones at dorsal and volar sides, Figure 5-e. SLAM method was simulated by drilling the scaphoid and lunate on the lateral side along the coronal plane. The position of the tunnel is from midlateral side of the scaphoid to the proximal ulnar corner of the lunate; the tendon graft is passed through the scaphoid and anchored to the lunate connecting the central regions of both bones, Figure 5-f. The stiffness of the tendon grafts in all cases was assumed to be the same as the stiffness of the dorsal SLIL [21].

In the FE models for the virtual reconstruction techniques, the holes in the bones were produced by positioning an axis linking the insertion points at dorsal and volar sides; then a cylinder with the same diameter as the bone graft was aligned to the axis. The cut to extract the cylindrical portion from the bone was achieved using a Boolean operation. The tendon graft representations were assumed to be fully bounded (tie-constraint) to the internal surfaces of the bone.The solver approach used was static/general. Verification was performed by comparing the resultant forces at the bottom surfaces of the radius and ulna with the applied loads. 


\section{SL Gap and Angle Calculation}

The SL gap was calculated at volar and dorsal side for the type II wrist from the models. The SL gap was calculated as the distance between two points on the edges of lunate and scaphoid at both dorsal and volar side. To locate the points to measure the SL gap, two imaginary arcs, Gilula lines, were used (Figure 6-a): a proximal arc, running along the proximal convexities of the scaphoid, lunate and triquetrum and a distal arc, tracing the distal curvatures of the scaphoid, lunate and triquetrum. An auxiliary arc was then drawn at the mid-point between the proximal and distal arcs lines, the intersection between the auxiliary arc and the edges of the scaphoid and lunate was employed to define the points used to measure the SL gap; $\mathrm{S}_{\mathrm{D}}$ $\mathrm{L}_{\mathrm{D}}$ for dorsal side and $\mathrm{S}_{\mathrm{V}}-\mathrm{LV}_{\mathrm{V}}$ for volar side (Figure 6-a). The SL angle was calculated using two lines projected on a lateral view using the method described by Larsen et al [32]; on the scaphoid, a line tangential to the proximal and distal margins at volar side; on the lunate, a line perpendicular to the tangent of the two distal poles, Figure 6-b.

In addition, to further facilitate comparison of the performance of the reconstruction techniques, the area affected by the rupture of SL ligament was analysed by obtaining contact area values and contact patterns for the articular cartilage surface between the scaphoid and lunate for the intact ligament case, SLIL sectioning and the three virtual reconstructions scenarios.

In order to assess the motion of the carpal bones, the position of each bone was obtained from the numerical models for all the scenarios at neutral and ulnar deviated clenched fist positions. Each bone was represented as a point located at its volume centroid. The coordinates of each bone centroid were plotted for the two positions to visualise the motion in three orthogonal planes.

\section{RESULTS}

Reconstruction techniques comparison: Dorsal and volar SL gap and angle

The predicted values for SL gap for all the scenarios including the reconstruction techniques are presented in Figure 7-a for the type II wrist. Results are shown next to corresponding predictions for a type I wrist, obtained from a previous study by the authors [21]. For the intact scenario in neutral position, the SL gaps predicted by the type II wrist model were 2.4 $\mathrm{mm}$ and $2.5 \mathrm{~mm}$ at dorsal and volar side respectively. For the ulnar deviated clenched fist 
position, the SL increased to $2.8 \mathrm{~mm}$ at both dorsal and volar side, an increase of $16.6 \%$ and $12.0 \%$, respectively, compared to the neutral position.

Following SLIL sectioning, in ulnar deviated clenched fist position, the SL gap increased by $0.7 \mathrm{~mm}(25 \%)$ and $0.3 \mathrm{~mm}(10.7 \%)$ at dorsal and volar side compared to the intact scenario. At the dorsal side, all three reconstruction techniques reduced the SL gap by $0.8 \mathrm{~mm}(20 \%)$ compared to the SLIL sectioning, to $0.1 \mathrm{~mm}(3.5 \%)$ less than that of the intact scenario. At volar side, the Corella and the SLAM techniques both reduced SL gap compared to SLIL sectioning, by $0.5 \mathrm{~mm}(16 \%)$ and $0.7 \mathrm{~mm}(22.6 \%)$, respectively, to $0.2 \mathrm{~mm}(7.1 \%)$ and 0.4 $\mathrm{mm}(14.2 \%)$ lower respectively than the intact scenario. The MBT technique failed to reduce the volar SL gap following SLIL sectioning, with the gap remaining $0.3 \mathrm{~mm}(10.7 \%)$ greater than for the intact scenario.

Predicted values for SL angle for the type II wrist for the scenarios are presented in Figure 7b. As with SL gap, results are shown next to corresponding predictions for a type I wrist [21]. The SL angle from the intact scenario increased by $5.9 \%$ from $42.3^{\circ}$ at neutral position to $44.8^{\circ}$ at the ulnar deviated clenched fist position. After SLIL sectioning, SL angle increased by $1.1^{\circ}(2.5 \%)$. The reconstruction techniques all reduced SL angle compared to the SLIL sectioning case, to below the intact value: Corella, SLAM and MBT techniques reduced SL angle to below the intact ulna deviate clenched value by $3.8^{\circ}(8.4 \%), 4.3^{\circ}(9.6 \%)$ and $5^{\circ}$ $(11.2 \%)$ respectively.

Contact area: scaphoid - lunate bones

We compared scaphoid/lunate contact area for our type I and II intact wrist models with experimentally determined values available in the literature [33-35]. Figure 8 shows the scaphoid/lunate contact area ratios predicted by our type I and II intact wrist models alongside the values from the cadaveric experimentation undertaken by Tencer et al [33], Viegas et al [34] and Blevens et al [35]. Note, the values for the type I wrist were not previously reported [21] but have been calculated as part of the current study. Upon inspection of Figure 8 it can be seen that overall, our model predictions compare well with the experimental determined values. 
Figure 9 shows the contact area values between the scaphoid and lunate for the various scenarios for both the type I (Figure 9-a) and type II wrist (Figure 9-b). For the type I wrist, Figure $9-\mathrm{a}$, in the intact ulnar deviated clenched fist case, a relatively small region $\left(1.2 \mathrm{~mm}^{2}\right)$ of scaphoid-lunate contact area can be discerned towards the dorsal side of the lunate. Following SLIL sectioning, there is no contact at all between the scaphoid and the lunate. The Corella reconstruction technique restored connection between the scaphoid and lunate, resulting in a contact region of similar shape, location and area $\left(1.7 \mathrm{~mm}^{2}\right)$, to the intact wrist ulnar deviated clenched fist case. The MBT technique failed to restore any contact between the scaphoid and lunate, whereas SLAM resulted in increased contact between the scaphoid and lunate, resulting in a contact region of greater area $\left(3.8 \mathrm{~mm}^{2}\right)$ being produced towards the location of the hole drilled in the scaphoid through which the graft passes.

For the type II wrist, Figure 9-b, in the intact ulnar deviated clenched fist case a region (5.0 $\mathrm{mm}^{2}$ ) of scaphoid-lunate contact can be seen towards the dorsal side of the lunate. Following SLIL sectioning, a small contact region remains $\left(1.4 \mathrm{~mm}^{2}\right)$ between scaphoid and lunate. Corella results in the connection between the scaphoid and lunate being restored, resulting in a contact region of similar shape, location and area $\left(6.9 \mathrm{~mm}^{2}\right)$ to the intact wrist ulnar deviated clenched fist case. As with the Type I wrist, SLAM results in increased contact between the scaphoid and lunate, medially $\left(14.8 \mathrm{~mm}^{2}\right)$, around the scaphoid graft hole, compared to the intact $\left(5 \mathrm{~mm}^{2}\right)$. Of the three techniques, MBT resulted in scaphoid-lunate contact area $\left(5.2 \mathrm{~mm}^{2}\right)$ closer to the intact case.

\section{Type I and II carpal mechanics: row/column theory}

Figure 10 shows the motion pattern of the bones of the carpal joint for both the type I and type II wrist when moving from the neutral to ulnar deviated clench fist position; superior, volar and lateral views are presented.

For the type I wrist, the plots show that scaphoid and lunate displacement is relatively small during the motion. The scaphoid and lunate exhibit similar displacement in two planes (ZX and ZY); in plane ZX both bones displaced $1.2 \mathrm{~mm}$ while in plane ZY scaphoid displaced $1.5 \mathrm{~mm}$ and lunate $1.6 \mathrm{~mm}$; scaphoid displacement is greater compared to the lunate in the $\mathrm{YX}$ plane, $1.3 \mathrm{~mm}$ and $0.9 \mathrm{~mm}$ respectively. Triquetrum and Pisiform displacement is similar in all three planes and of greater magnitude than the scaphoid and lunate. The displacement of the trapezoid, trapezium, capitate and hamate is appreciably greater than that of the scaphoid and lunate, in addition, these bones exhibit a similar displacement pattern. 
For the type II wrist, the plots indicate that as the wrist moves from the neutral to ulnar deviated clench fist position the triquetrum and pisiform displace dorsally, exhibiting similar displacement in the 3 planes. In comparison, scaphoid displacement is smaller in the volar and distal directions. The trapezoid, trapezium, capitate, hamate and lunate all exhibit a similar motion pattern, with displacement less than the triquetrum, pisiform and scaphoid. Whilst scaphoid and lunate displacement is similar in the YX plane $(1.1 \mathrm{~mm}$ and $1.2 \mathrm{~mm}$, respectively), differences can be clearly seen in the ZX where scaphoid moved $0.6 \mathrm{~mm}$ and lunate $1.2 \mathrm{~mm}$; and ZY planes where scaphoid moved $0.9 \mathrm{~mm}$ and lunate $0.7 \mathrm{~mm}$.

\section{Type I and II performance of the reconstruction techniques: wrist kinematics}

Figures 11-14 show the position of the carpal joint bones for the intact wrist compared to SLIL sectioning, Corella, SLAM and MBT technique application, for the type I (Figures 1112) and type II wrist (Figures 13-14) in the ulnar deviated clench fist position; superior, volar and lateral views are presented. Also shown in Figures 11-14 is a tabular comparison of the position of the centroid of each of the carpal joint bones for SLIL, sectioning, Corella, SLAM and MBT techniques compared to the intact wrist for the ulnar deviated clench fist position.

A comparison of the techniques, Table 1 shows the distance calculated between the points representing the centroids of the scaphoid and lunate bones, following SLIL sectioning and Corella, SLAM and MBT techniques compared to the intact wrist case for both the type I and type II wrist in the ulnar deviated clenched fist position. The Finite Element Analysis software, Abaqus, provided the coordinates of the centroids of the bones, facilitating their tracking during the movement.

\section{Type I Wrist}

It can be seen upon inspection of Figure 11-a that after SLIL sectioning of the type I wrist, the scaphoid exhibits greater displacement in the three planes compared to the lunate (2.05mm overall compared to $1.62 \mathrm{~mm})$. Displacement of the lunate is similar to that of the intact case, only $1.9 \%$ greater overall, whereas scaphoid displacement is $22.8 \%$ greater compared to the intact wrist. The distance between the scaphoid and lunate centroids increased by $1.2 \mathrm{~mm}$ compared to the intact wrist as a result of SLIL sectioning (Table 1). Following application of the Corella technique (Figure 11-b), the scaphoid and lunate displacement pattern was similar to that of the intact scenario (lunate displacement $0.63 \%$ lower, scaphoid $3.6 \%$ greater), with the distance between the centroids of the two bones 
being slightly smaller compared to the intact case, $(0.1 \mathrm{~mm}$ less, Table 1$)$. No significant change in the motion of the other carpal bones was discernible. For the SLAM technique (Figure 12-a), scaphoid and lunate displacement pattern diverged from that of the intact (lunate displacement $6.9 \%$ lower, scaphoid $12 \%$ greater). The distance between the scaphoid and lunate was greater compared to the intact case (by $0.7 \mathrm{~mm}$, Table 1), with the lunate exhibiting displacement more to the volar side than the intact case with the scaphoid displacing towards the proximal pole, at the articulation area with the scaphoid. After MBT reconstruction (Figure 12-b), the scaphoid and lunate displacement is similar in nature to the intact case, with the distance between the centroids of the two bones being greater compared to the intact case (by $0.37 \mathrm{~mm}$, Table 1) but smaller compared to the SLAM technique. No significant change compared to the intact wrist in the displacement pattern of the remaining carpal bones was observed following MBT reconstruction simulation.

\section{Type II Wrist}

Following SLIL sectioning of the type II wrist (Figure 13-a), both scaphoid and lunate movement remains close to that of the intact, displacement of the two bones is slightly greater $(0.1 \mathrm{~mm}$ lunate, $0.4 \mathrm{~mm}$ scaphoid) but the relative position of the two bones remains very similar to the intact ligament case, with scaphoid - lunate centroid distance increasing by only $0.24 \mathrm{~mm}$ in comparison (Table 1 ).

Following application of the Corella technique (Figure 13-b), the displacement of the trapezium, trapezoid, capitate and hamate remained similar to that of the intact case, but lunate displacement was altered as a consequence of gap over-closure after applying the reconstruction technique. In addition, the scaphoid moves slightly more towards the lunate, however the direction of the motion for both the scaphoid and lunate is broadly similar to the intact case but with the magnitude being greater for both bones $(0.1 \mathrm{~mm}$ scaphoid, $0.3 \mathrm{~mm}$ lunate). For the Corella technique, scaphoid-lunate centroid distance was $0.21 \mathrm{~mm}$ less than the intact case (Table 1). Following application of the SLAM technique (Figure 14-a), scaphoid displacement was more towards the lunate compared to the intact case, with the scaphoid taking a more vertical position during the movement to the ulnar deviated clench fist position. Compared to the intact wrist, both scaphoid and lunate motion was greater $(0.4 \mathrm{~mm}$ scaphoid, $0.2 \mathrm{~mm}$ lunate). The motion of the trapezium, trapezoid, capitate and hamate and of the triquetrum and pisiform was not significantly altered following application of the technique. Lunate displacement tended to be more in the lateral direction with the 
distance between the lunate and scaphoid bones being discernibly less (by $1 \mathrm{~mm}$, Table 1), following SLAM application compared to the intact scenario. After MBT reconstruction (Figure 14-b), lunate motion pattern is altered due to the constraint of the ligament graft at the dorsal side. The distance between the centroids of the lunate and scaphoid bones is larger compared to the intact scenario $(18.88 \mathrm{~mm}$ compared to $18.81 \mathrm{~mm}$, Table 1$)$.

Comparing the distances presented in Table 1, it can be seen that for the type I wrist, Corella reconstruction resulted in a lunate-scaphoid centroid distance closest to the intact wrist (within $0.1 \mathrm{~mm}$ ), followed by MBT then SLAM. For the type II list, MBT reduced lunatescaphoid distance closer to the intact wrist (within $0.07 \mathrm{~mm}$ ), followed by Corella and SLAM.

\section{DISCUSSION}

Reconstruction techniques comparison: dorsal and volar SL gap and angle \& contact area analysis

The results of the reconstruction technique comparison indicates that for the type II wrist, the Corella reconstruction technique is better able to restore dorsal and volar gap and SL angle to that of the intact (ligament) wrist following SLIL sectioning than the SLAM and MBT techniques. Application of the Corella technique following simulated SLIL sectioning, resulted in a dorsal gap within 3.5\%, a volar gap within $7.1 \%$ and an SL angle within $8.4 \%$ of the intact scenario. Of the two other techniques considered, SLAM was better able to restore dorsal, volar gap and SL angle than MBT. The authors' previous investigation also found that the Corella technique was the most effective reconstruction method, better able to restore SL gap and angle closer to that of the intact ligament for type I wrists, followed by SLAM. The results from the contact area analysis for the type II and I wrists presented support the SL gap and angle findings. The Corella technique produced scaphoid-lunate contact patterns and areas of similar shape and values to those of the intact wrist.

Following SLIL sectioning, our study determined there was no contact area between scaphoid-lunate cartilage articulation for the type I wrist, whereas for the type II wrist, some contact was maintained. This supports the findings of previous clinical and cadaveric research that determined that the extra articulation in a type II wrist may help to reduce instability in cases of SL ligament injury [4-6]. 
Our model predicted SL gaps in the range 1.7-2.7mm for Corella, 2.1-2.7 for SLAM and 2.23.1 for MBT compared to 1.8-2.8 for the intact case. The relatively small differences in the SL gap between the different reconstruction techniques predicted by our model concur with the results from cadaver experimentation and reported clinical outcomes. Lee et al [20] report the results of the application of three scapholunate reconstruction techniques (SLAM, MBT and Blatt capsulodesis) in cadaver limbs. Both the MBT and SLAM techniques were found to restore SL gap to within $1 \mathrm{~mm}$ of the intact case for a clenched fist posture. In a retrospective cohort study, Links et al [36] compared clinical and radiographic outcomes in patients with chronic scapholunate dissociation following treatment with MBT versus 4-bone tendon weave technique. In the case of MBT the SL gap following reconstruction was found to be $2.2 \pm 0.4 \mathrm{~mm}$. In another study of 19 patients who had undergone the MBT procedure, Chabas et al [37] reported a mean static SL distance of $2.4 \mathrm{~mm}$. Yao et al [38] report the outcome for 13 patients who had undergone SLAM, after a follow-up period of 11 months the mean postoperative scapholunate gap was $2.1 \mathrm{~mm}$. Although relatively small, research indicates that these differences can be clinically significant. Ideally, the surgical technique should restore a normal relationship between carpal bones, preserve range of motion and grip strength whilst achieving a pain free wrist. Further, unsatisfactory results have been reported for techniques that fail to directly correct SL gap [37]. Where clinical outcomes have been compared, greater improvement in mean pain and DASH scores, motion and grip strength have been reported for techniques that were able to provide relatively small (e.g $2.2 \pm 0.4 \mathrm{~mm}$ compared to $3.0 \pm 0.8 \mathrm{~mm}$ ) yet significantly greater improvement of scapholunate gap and angle [38].

\section{Type I and II carpal mechanics: row/column theory}

Several previous studies have attempted to elucidate the row and column theories of carpal mechanics and relate them to type I and II wrists $[8,11,13]$. The results from our study support the assertion that type I wrists exhibit row mechanics and type II column mechanics $[8,11]$. The analysis of the motion pattern of the bones of the carpal joint for the type I wrist when moving from the neutral to ulnar deviated clench fist position (Figure 2-b) shows that the lunate rotates clockwise to the ulnar side sliding over the radius; at the lateral side of the lunate, the scaphoid follows the motion preserving the distance between the bones and hence 
their relative position in two planes (ZX and ZY, Figure 10) as described by row theory. In the YX plane, the displacement of the scaphoid is greater; as a consequence of the rotation of the scaphoid to a more vertical position. The remaining bones which are considered to make up the proximal row, the triquetrum and pisiform, exhibit a similar displacement pattern to each other. In addition, the analysis shows that trapezium, trapezoid, capitate and hamate (distal row) move together, as a lever unit rotating around a centre, the capitate being the closest bone to the pivot point; during the motion, the trapezium and trapezoid moves upwards following the rotation whilst the capitate, and the hamate move downwards. This unit (the distal row) behaves like a rigid unit, with the distance between the bones being preserved in all three planes during motion.

For the case of the type II wrist, the trapezium, trapezoid, capitate, hamate and lunate exhibited similar motion pattern, behaving as a unit (central column) rotating around the lunate. The proximal bones of the type II wrist, the scaphoid, lunate and triquetrum exhibited displacement patterns which were clearly different from each other, which was not the case for the type I wrist, supporting the view that these three bones belong to three distinct columns, the lateral, central and medial columns respectively. Unlike the type I wrist, for type II, the scaphoid displacement is similar to that of the lunate only in the YX plane (Figure 10); in planes ZX and ZY, the magnitude of the displacement of the scaphoid is different as a consequence of a rotation along the $\mathrm{X}$-axis to take up a more vertical position and the rotation around its longitudinal axis. The triquetrum and pisiform demonstrated greater displacement compared to other bones especially in the YX plane. This is in accordance with column theory, whereby during the movement, the medial column (triquetrum and pisiform) tilts dorsally to make space for the bones of the central column, this movement affects the relative position between the lunate and the triquetrum in the ZX and ZY planes.

\section{Type I and II performance of the reconstruction techniques: wrist kinematics}

Our analysis of wrist kinematics following application of the ligament reconstruction simulations to the type I wrist indicated that none of the techniques significantly altered the motion pattern of the wrist, all continued to exhibit row behaviour. Following simulation of the Corella reconstruction, motion of the bones in the distal row was effectively unchanged, the triquetrum behaved as in the intact scenario; the lunate and scaphoid exhibited similar motion pattern as the intact case but maintaining slightly closer proximity to each other due to the constraints at both volar and dorsal sections. In the case of SLAM reconstruction, in the 
proximal row, the triquetrum maintained the motion pattern as in the intact wrist case. Compared to the intact wrist, the lunate moved more volarly, in the centre where the bone is attached to the scaphoid by the ligament reconstruction. Scaphoid motion was more towards the proximal pole at the articulation area to the scaphoid, where the two bones are linked by the ligament after the reconstruction. The distance between the two bones was greater compared to the intact case. For MBT, the motion of the bones in the distal row following reconstruction was akin to that of the intact wrist, as was the triquetrum motion (proximal row). The lunate and scaphoid moved together similar to the intact but sliding slightly more over the radius. Again, the distance between the lunate and scaphoid bones was greater compared to the intact case. Overall, the kinematic analysis confirmed that the Corella technique is best able to restore motion closest to the intact scenario for the type I wrist.

For the type II wrist, the kinematic analysis also determined that application of the ligament reconstruction techniques does not significantly alter wrist motion pattern (column behaviour). Following application of Corella reconstruction, motion of the trapezium, trapezoid, capitate and hamate (central column) remained essentially unchanged compared to the intact wrist, in addition, the direction of motion of the bones of the medial column (triquetrum and pisiform) was unaltered. The scaphoid (lateral column) motion was slightly more towards the lunate (central column) due to the constraints imposed at dorsal and volar side by the ligament graft. For the SLAM technique, the only significant difference compared to the intact wrist was that the lateral column (scaphoid) moved more towards the central column (lunate), also, the scaphoid tended to take a more vertical position during the motion as a result of the insertion point of the ligament graft in the middle of the area that articulates to the lunate, allowing a point of rotation for this bone. In addition the lunate rotation was greater compared to the intact wrist as it followed the motion of the scaphoid as a result of the ligament graft. For the MBT reconstruction the motion of the bones of the central column did not change significantly compared to the intact wrist as was the case with the lateral column (scaphoid). In the central column, the lunate motion pattern was altered, at dorsal side the movement was constrainted by the ligament graft but at volar side the lack of a constraint, enabled a small rotation of the bone opening the gap at volar side. The medial column (triquetrum and pisiform) was also altered slightly, motion was greater as the column followed the motion of the lunate. Overall, the kinematic analysis confirmed that the Corella technique is best able to restore motion closest to the intact scenario for the type II wrist. 
The wrist kinematic analysis presented in the current study compared carpal bone motion of both type I and type II wrists following SLIL sectioning with the intact ligament scenario. For the type I wrist, following sectioning the scaphoid exhibited appreciably greater displacement whereas the lunate was less affected. For the type II wrist, however, both scaphoid and lunate motion remained very close to that of the intact. This finding supports those reported by Rhee [5] and Hasse [6], that type II wrists are less prone to instability even following scapholunate ligament injury.

Our model is subject to a number of simplifications, assumptions and limitations typical of complex numerical problems in this research field. For example, our model considered cancellous bone as exhibiting elastic-plastic, isotropic material behaviour. The intact wrist model developed using these properties has been successfully validated previously against the results from cadaveric experimentation [21] so the material properties employed can be considered sufficiently accurate for this investigation. Also, while it is recognized that the elastic modulus of cancellous bone depends primarily on apparent bone density, the exact form of the dependency is contested [39] and this is further complicated by the dependency of the relationship on trabecular orientation, loading direction and anisotropy. However, it is recognised that within a single anatomical site density range is limited, [39] which suggests that an invariant bone property, as was employed in our study, should provide sufficient accuracy.

The majority of the ligaments included in the model were represented using one-dimensional spring elements, which although a common approach, is known to have its limitations, for example, in cases of complex non uniform 3-D stress/strain [40]. More accurate representation requires 3-D Finite Element modelling treatment, however the required approach is highly involved and extremely time consuming [40].We assumed linear elastic material properties for the ligaments in the model where in practice ligaments tend to exhibit non linear viscoelastic behaviour. However, a large number of parameters are required for accurate non linear viscoelastic behaviour representation, therefore parameter data availability is key but this data is generally not widely available [40]. In practice though, ligaments tend to operate in or close to the linear region, so our modelling assumption should provide reasonable accuracy [41]. A hyperelastic material model was employed to represent soft tissue (cartilage) behaviour, which is considered to be an accurate approach [29]. 
Finally, the type II wrist models were developed from the CT scan of the left hand of a single subject, therefore any results and conclusions drawn from the analyses should be viewed in this context and interpreted with care. Future planned work includes analysis of additional subjects to provide further potential support and verification for the findings of the current investigation. In conclusion, our study has determined that the Corella ligamentous reconstruction technique is best able to restore SL gap, angle and stability following scapholunate ligament injury for both type II and type I wrists and is able to do so without altering the kinematics of the wrist. Our findings also support the view that in term of wrist mechanics, type I wrists exhibit row behaviour and type II wrists column behaviour. In addition, our analysis suggests that the extra articulation between the lunate and hamate in a type II wrist may help to improve stability of the wrist following scapholunate ligament injury. 


\section{REFERENCES}

1. Kitay A, Wolfe SW. Scapholunate Instability : Current Concepts in Diagnosis and Management. J Hand Surg Am. 2012;37(10):2175-96.

2. Andersson JK. Treatment of scapholunate ligament injury. EFORT Open Rev. 2017;2:382-93.

3. Jones, W.A. Beware the sprained wrist. The incidence and diagnosis of scapholunate instability. J Bone Joint Surg Br. 1988;70:293-7.

4. Werner FW, Short WH, Green JK, Evans PJ, Walker JA. Severity of Scapholunate Instability Is Related to Joint Anatomy and Congruency. J Hand Surg Am. 2007;32(1):55-60.

5. Rhee PC, Moran SL, Shin AY. Association Between Lunate Morphology and Carpal Collapse in Cases of Scapholunate Dissociation. J Hand Surg Am. 2009;34(9):1633-9.

6. Haase SC, Berger RA, Shin AY. Association between lunate morphology and carpal collapse patterns in scaphoid nonunions. J Hand Surg Am. 2007;32(7):1009-12.

7. Pang EQ, Douglass N, Kamal RN. Association of Lunate Morphology With Carpal Instability in Scapholunate Ligament Injury. Hand. 2017;1-5

8. Galley I, Bain GI, McLean JM. Influence of Lunate Type on Scaphoid Kinematics. J Hand Surg Am. 2007;32(6):842-7.

9. Viegas SF, Wagner K, Patterson R, Peterson P. Medial (hamate) facet of the lunate. J Hand Surg Am. 1990;15(4):564-71.

10. Morimoto H., Goto A., Sato Y. et al. The triquetrum-hamate joint: An anatomic and in vivo three-dimensional kinematic study. J Hand Surg Am. 2003; 28(5): 797-805.

11. Craigen MAC, Stanley JK. Wrist kinematics. Row, column or both? J Hand Surg Am. 1995;20(2):165-70.

12. Viegas SF, Patterson RM, Hokanson JA, Davis J. Wrist anatomy: Incidence, distribution, and correlation of anatomic variations, tears, and arthrosis. J Hand Surg Am. $1993 ; 18(3)$. 
13. Nakamura K, Beppu M, Patterson RM, Hanson CA, Hume PJ, Viegas SF. Motion analysis in two dimensions of radial-ulnar deviation of type I versus type II lunates. J Hand Surg Am. 2000;25(5).

14. McLean JM, Turner PC, Bain GI, Rezaian N, Field J, Fogg Q. An association between lunate morphology and scaphoid-trapezium-trapezoid arthritis. J Hand Surg Eur Vol. 2009;34(6):778-82.

15. Ferris BD, Stanton J, Zamora J. Kinematics of the Wrist. J Bone Jt Surg. 2000;(82B):242-5.

16. Brunelli GA, Brunelli GR. A new technique to correct carpal instability with scaphoid rotary subluxation: a preliminary report. J Hand Surg. 1995;20:S82-S85.

17. Garcia-Elias M, Lluch AL, Stanley JK. Three-ligament tenodesis for the treatment of scapholunate dissociation: indictions and surgical technique. J Hand Surg. 2006;31:125-134.

18. Van Den Abbeele KL, Loh YC, Stanley JK Trail IA. Early results of a modified Brunelli procedure for scapholunate instability. J Hand Surg. 1998;23:258-261.

19. Corella F., Del Cerro M, Ocampos M, Larrainzar-Garijo R. Arthroscopy ligamentoplasty of the dorsal and volar portions of the scapholunate ligament. J Hand Surg. 2014;39:643-650.

20. Lee SK, Zlotolow DA, Sapienza A, Karia R, Yao J. Biomechanical comparison of 3 methods of scapholunate ligament reconstruction. J Hnd Surg. 2014;39:643-650.

21. Alonso Rasgado T, Zhang Q, Jimenez Cruz D, Bailey C, Pinder E, Mandaleson A, et al. Analysis of tenodesis techniques for treatment of scapholunate instability using the finite element method. Int j numer method biomed eng. 2017;33(12):1-12.

22. Fischli S, Sellens RW, Beek M, Pichora DR. Simulation of extension, radial and ulnar deviation of the wrist with a rigid body spring model. J Biomech. 2009;42(9):1363-6.

23. Gíslason MK, Stansfield B, Nash DH. Finite element model creation and stability considerations of complex biological articulation: The human wrist joint. Med Eng Phys. 2010;32(5):523-31. 
24. Kijima Y, Viegas SF. Wrist Anatomy and Biomechanics. J Hand Surg Am. 2009;34(8):1555-63.

25. Berger RA. The Anatomy and Basic Biomechanics of the Wrist joint. J Hand Ther. 1996;9(2):84-93.

26. Nagao S, Patterson RM, Buford WL, Andersen CR, Shah MA, Viegas SF. Threedimensional description of ligamentous attachments around the lunate. J Hand Surg Am. 2005;30(4):685-92.

27. Nakamura K, Patterson RM, Moritomo H, Viegas SF. Type I versus type II lunates: Ligament anatomy and presence of arthrosis. J Hand Surg Am. 2001;26(3):428-36.

28. Berger RA, Imeada T,Berglund L, An KN. Constraint and material properties of the subregions of the scapholunate interosseous ligament. J Hand Surg AM. 199;24:953-962.

29. Bajuri MN, Abdul Kadir MR, Murali MR, Kamarul T. Biomechanical analysis of the wrist arthroplasty in rheumatoid arthritis: a finite element analysis. Med Biol Eng Comput. $2012 ; 1-12$.

30. Savelberg HH, Kooloos JG, Huiskes R, Kauer JM. Stiffness of the ligaments of the human wrist joint. J Biomech. 1992;25:369-376.

31. Guo X, Fan Y, Li ZM. Effects of dividing the transverse carpal ligament on the mechanical behavior of the carpal bones under axial compressive load: A finite element study. Med Eng Phys. 2009;31(2):188-94.

32. Larsen CF, Stigsby B, Lindequist S, Bellstrøm T, Mathiesen FK, Ipsen T. Observer variability in measurements of carpal bone angles on lateral wrist radiographs. J Hand Surg Am. 1991;16(5):893-8.

33. Tencer AF, Viegas SF, Cantrell J, Chang M, Clegg P, Hicks C, O'Meara C, Williamson JB. Pressure distribution in the wrist joint. J Orthop Res. 1988;6(4):509-17.

34. Viegas SF, Tencer AF, Cantrell J, Chang M, Clegg P, Hicks C, O'Meara C, Williamson JB. Load transfer characteristics of the wrist. Part I. The normal joint. J Hand Surg Am. 1987 Nov;12(6):971-8. 
35. Blevens AD, Light TR, Jablonsky WS, Smith DG, Patwardhan AG, Guay ME, Woo TS. Radiocarpal articular contact characteristics with scaphoid instability. J Hand Surg Am. 1989 Sep;14(5):781-90.

36. Links AC, Chin SH, Waitayawinyu T, Trumble TE. Scapholunate interosseous ligament reconstruction: results with a modified Brunelli technique versus four-bone weave. $\mathbf{J}$ Hand Surg Am. 2008 Jul-Aug;33(6):850-6. doi: 10.1016/j.jhsa.2008.02.010

37. Chabas JF, Gay A, Valenti D, Guinard D, Legre R. Results of the modified Brunelli tenodesis for treatment of scapholunate instability: a retrospective study of 19 patients. $\mathrm{J}$ Hand Surg Am. 2008 Nov;33(9):1469-77. doi: 10.1016/j.jhsa.2008.05.031.

38. Yao J, Zlotolow DA, Lee SK. ScaphoLunate Axis Method. J Wrist Surg. 2016 Mar;5(1):59-66. doi: 10.1055/s-0035-1570744. Epub 2016 Jan 6.

39. Keaveny TM, Morgan EF, Niebur GL, et al. 2001. Biomechanics of trabecular bone. Annu Rev Biomed Eng 3:307-333.

40. Weiss, J. A., J. C. Gardiner, B. J. Ellis, T. J. Lujan, and N. S. Phatak. (2005) Threedimensional finite element modeling of ligaments: Technical aspects. Med. Eng. Phys. 27(10):845-861.

41. Spratley, E. M., and J. S. Wayne. Computational model of the human elbow and forearm: Application to complex varus instability. (2011) Ann. Biomed. Eng. 39(3):10841091. 
TABLES

Table 1 Distance between centroids of the scaphoid and lunate bone

\begin{tabular}{|c|c|c|}
\hline & \multicolumn{2}{|c|}{ Distance [mm] } \\
\hline \multirow{2}{*}{ CASE } & $\begin{array}{c}\text { TYPE I } \\
\text { WRIST }\end{array}$ & $\begin{array}{c}\text { TYPE II } \\
\text { WRIST }\end{array}$ \\
\hline Intact & 21.26 & 18.81 \\
\hline SLIL & 22.46 & 19.05 \\
\hline Corella & 21.16 & 18.60 \\
\hline SLAM & 21.96 & 17.81 \\
\hline MBT & 21.63 & 18.88 \\
\hline
\end{tabular}

Supplementary Table. Ligaments included in the model

\begin{tabular}{|c|c|}
\hline Ligament & Stiffness (N/mm) \\
\hline Dorsal radiocarpal & $27[29]$ \\
\hline Radial collateral & 10 [29] \\
\hline Ulnar collateral & 100 [29] \\
\hline Radioulnar & 50 [29] \\
\hline Radioscaphocapitate & 50 [29] \\
\hline Long radiolunate & 75 [29] \\
\hline Short radiolunate & 75 [29] \\
\hline Ulnolunate & 40 [29] \\
\hline Ulnotriquetral & 40 [29] \\
\hline Radioulnar & 50 [29] \\
\hline Dorsal intercarpal & 128 [29] \\
\hline Dorsal trapeziotrapezoid & 110 [29] \\
\hline Dorsal capitotrapezoid & 300 [29] \\
\hline Dorsal capitohamate & 325 [29] \\
\hline Dorsal triquetrohamate & 300 [29] \\
\hline Dorsal lunatecapitate & 150 [29] \\
\hline Dorsal lunatehamate & $150[29]$ \\
\hline Dorsal scaphocapitate & 150 [29] \\
\hline Volar trapeziotrapezoid & 110 [29] \\
\hline Volar scaphotrapezial & 150 [29] \\
\hline Volar scaphotrapezoidal & 150 [29] \\
\hline Volar scaphocapitate & 40 [29] \\
\hline Volar capitotrapezoid & $80[22]$ \\
\hline
\end{tabular}




\begin{tabular}{|l|c|}
\hline \multicolumn{1}{|c|}{ Ligament } & Stiffness (N/mm) \\
\hline Volar capitohamate & $210[22]$ \\
\hline Volar triquetrocapitate & $40[29]$ \\
\hline Volar triquetrohamate & $300[29]$ \\
\hline Scapholunate Dorsal & $60[28,30]$ \\
\hline Scapholunate Volar & $30[28,30]$ \\
\hline Scapholunate Proximal & $15[27,29]$ \\
\hline Lunotriquetral Volar & $250[22]$ \\
\hline Trapeziotrapezoid & $110[21]$ \\
\hline Capitotrapezoid & $300[29]$ \\
\hline Capitohamate & $325[29]$ \\
\hline
\end{tabular}


Perform evaluation of surgical techniques for treatment of scapholunate instability in a type II wrist.

Novelty

- $\quad$ No previous studies have assessed the performance of scapholunate ligament reconstruction techniques considering the two types (I and II) of wrist.

- $\quad$ No previous studies have investigated wrist carpal mechanics both before and after disruption of the SL ligament based on lunate bone morphology. 


\begin{tabular}{|l|l|l|l|}
\hline \multicolumn{1}{|c|}{ Type II wrist } \\
Neutral position \\
\hline $\begin{array}{c}\text { Ligament } \\
\text { Interaction } \\
\text { at neutral } \\
\text { position }\end{array}$
\end{tabular}


Performance evaluation of surgical techniques for treatment of scapholunate instability in a type II wrist

Roberto Leonardo-Diaz, Teresa Alonso-Rasgado*, David Jimenez-Cruz, Colin Bailey, Sumedh Talwalkar

The performance of three tenodesis techniques used to repair scapholunate ligament disruption of a type II wrist was assessed using Finite Element based virtual surgery. The Corella technique restored dorsal gap, volar gap and scapholunate angle to within $3.5 \%, 7.1 \%$ and $8.4 \%$ respectively of the intact wrist. Application of the ligament reconstruction techniques did not significantly alter the motion pattern of the wrist. The extra articulation in a type II wrist may help to improve stability following scapholunate ligament injury. 


\section{Figures}

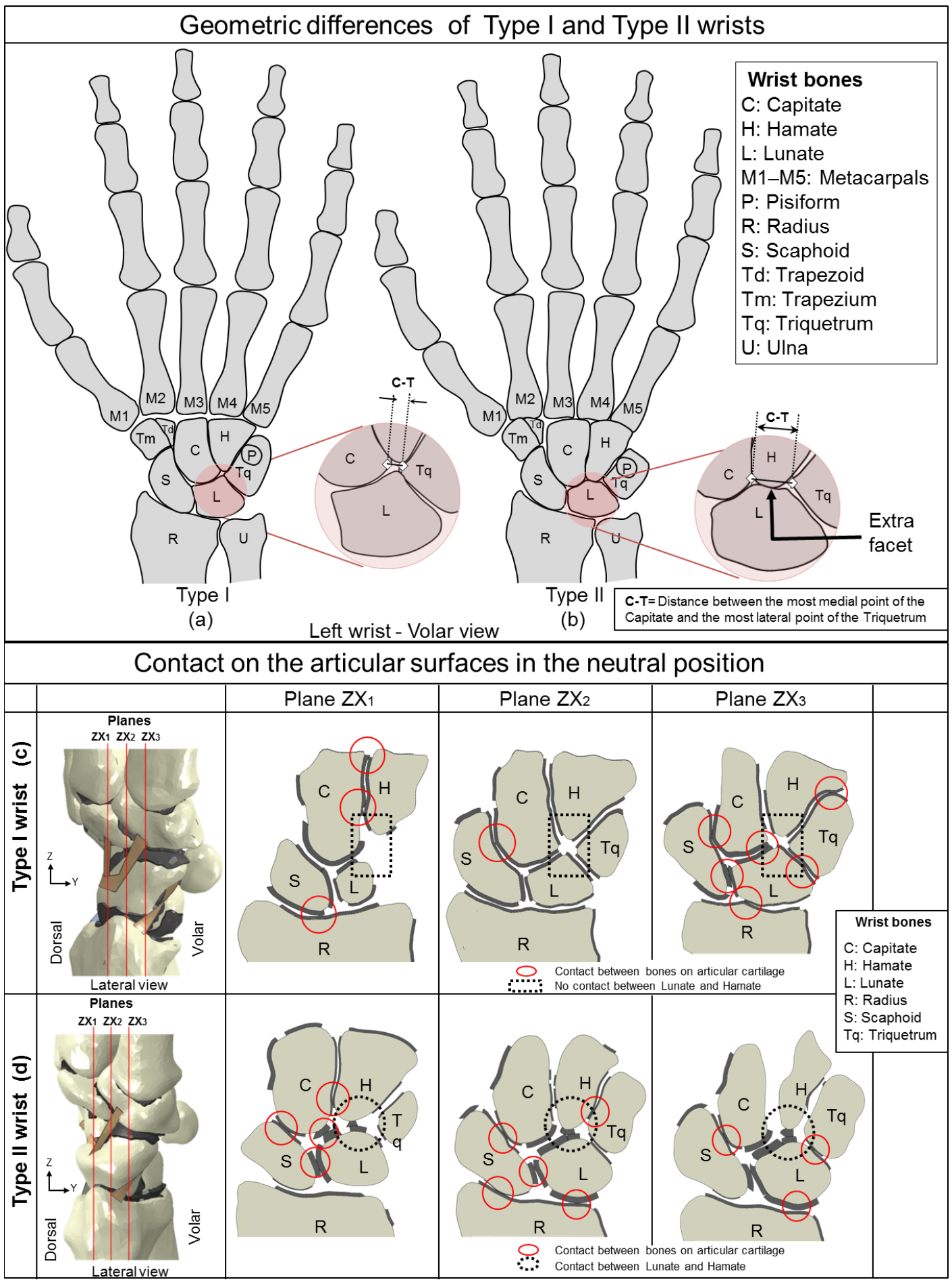

Figure 1 (a) Type I lunates do not articulate with hamate. (b) Type II lunates have an extra facet that allows articulation with the hamate, C-T distance ranging $\leq 4 \mathrm{~mm}$ to $8.9 \mathrm{~mm}$. (c) Contact between articular cartilages of the bones for different planes in the neutral position. Type I wrist, there is no contact between the hamate and lunate in any plane. (d) Type II wrist, there is contact between the hamate and lunate in more than one plane. 


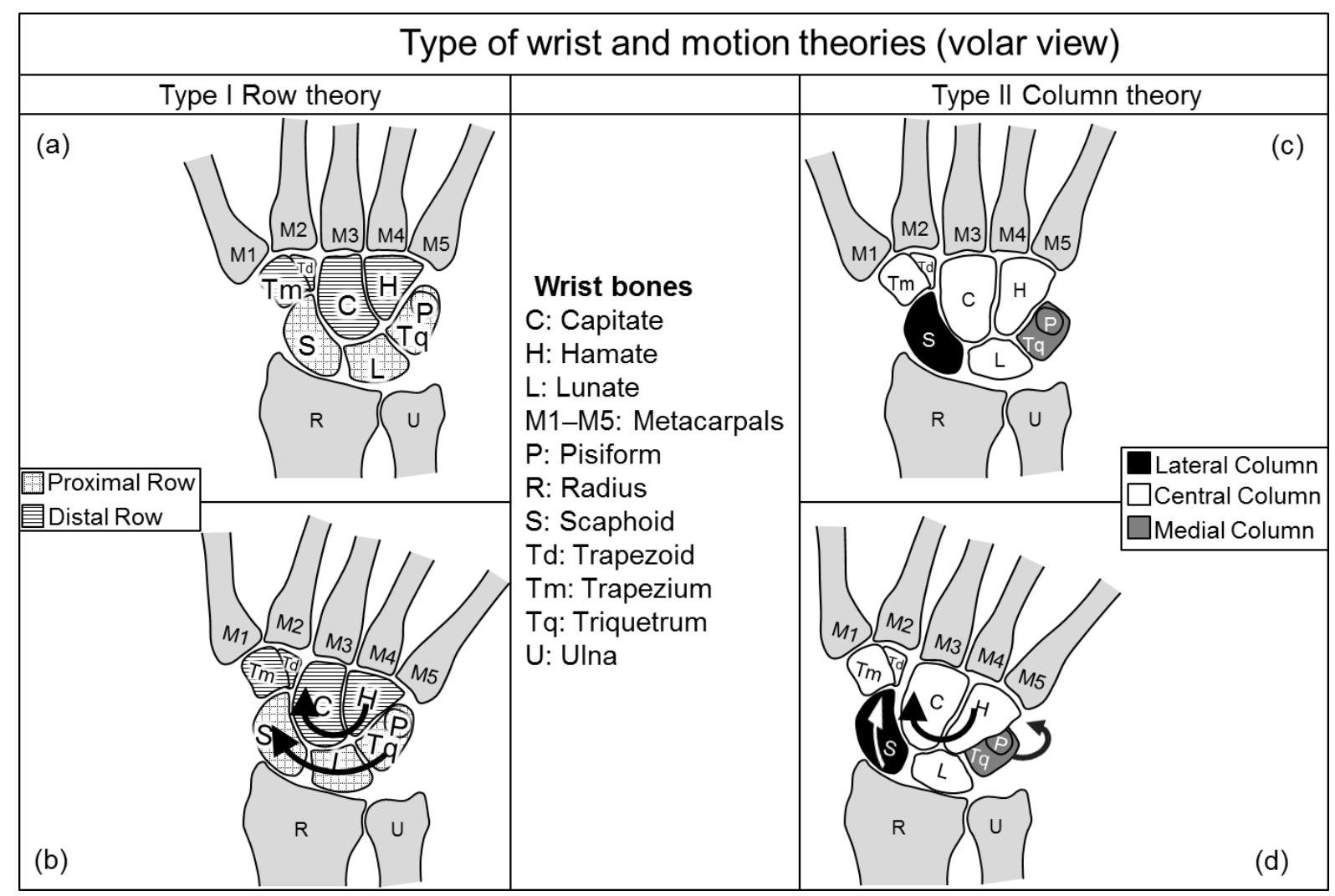

Figure 2 Wrist motion theories. Type I - Row theory; (a) Neutral position, (b) Ulnar deviated clench fist position. Type II - Column theory; (c) Neutral position, (d) Ulnar deviated clench fist position. 


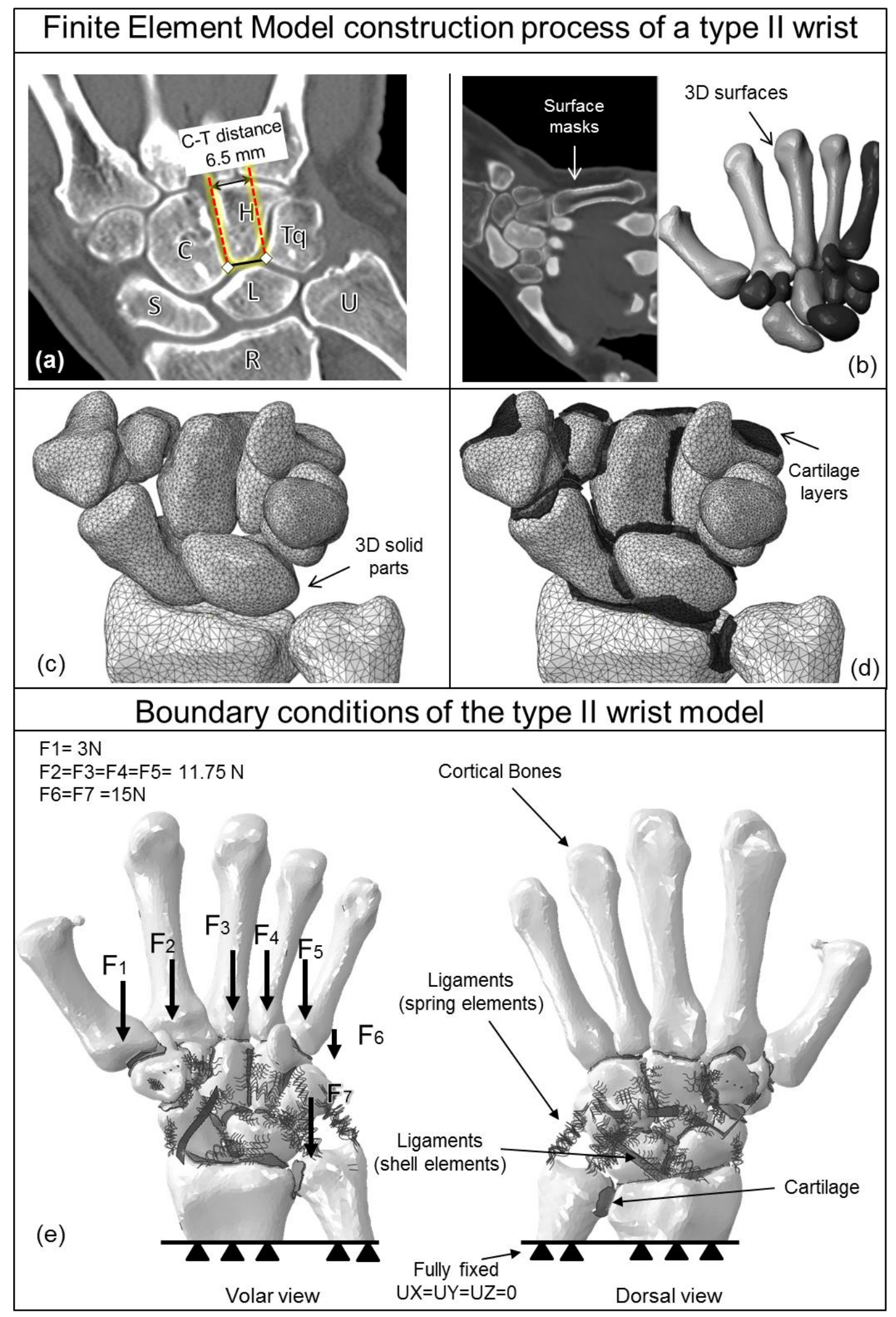

Figure 3 (a) CT scan, the distance between the capitate (C) and Triquetrum (Tq) is $6.5 \mathrm{~mm}$. (b) Masks drawn on the CT scan images using 3D image segmentation and processing software to generate the 3D surfaces. (c) Solid parts imported into Abaqus to create the assembly of the wrist. (d) Modelling of the cartilage by offsetting of elements at the articulating surfaces between bones. (e) Finite element model of the healthy wrist showing the bones, the articular cartilage, set of ligaments, forces and boundary conditions. 


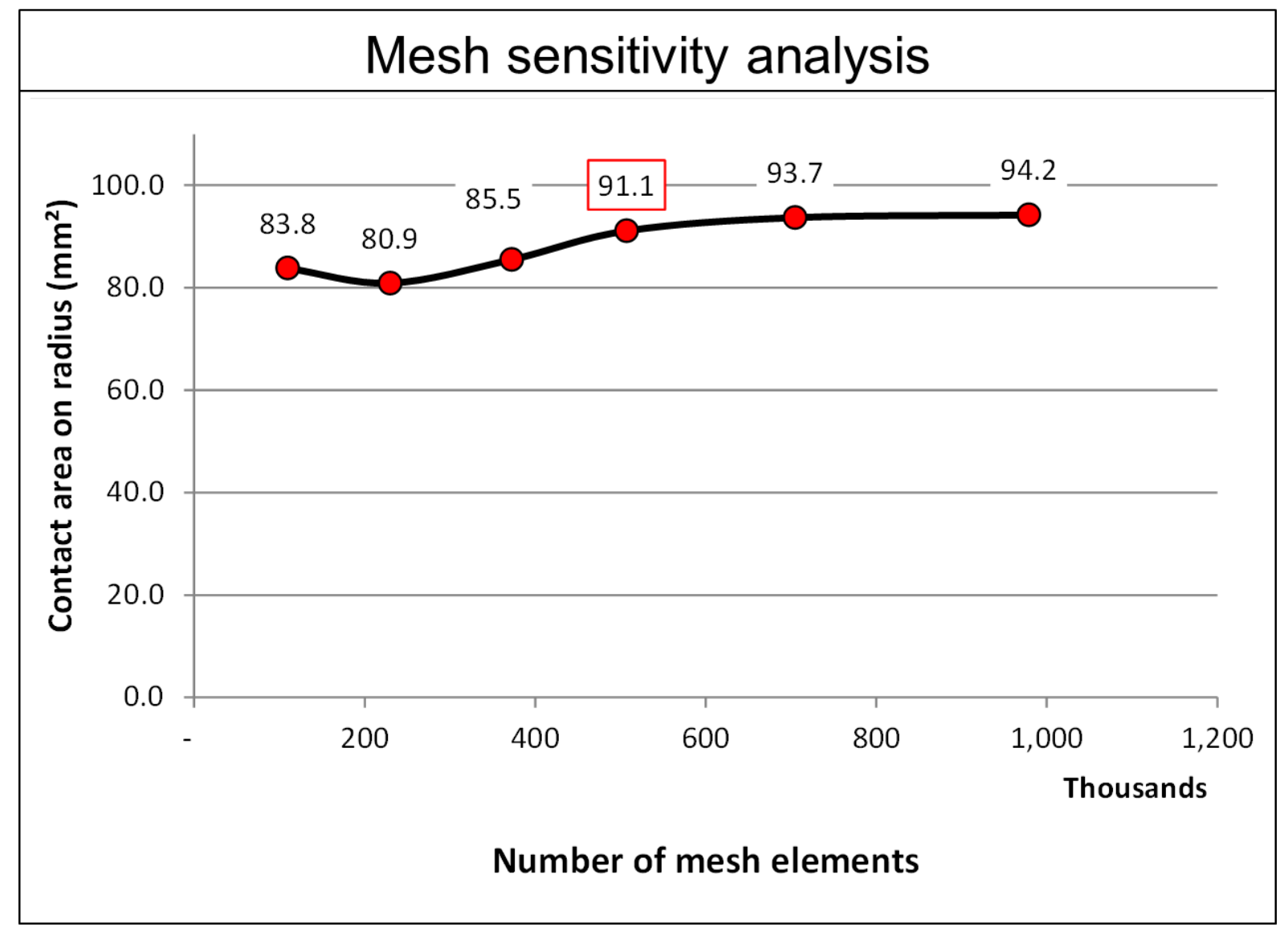

Figure 4 Mesh convergence plot. The contact area on radius converged when using a mesh with over 500,000 elements. In a model with 507,171 elements we were able to achieve accuracy in the results with less computing resource. 


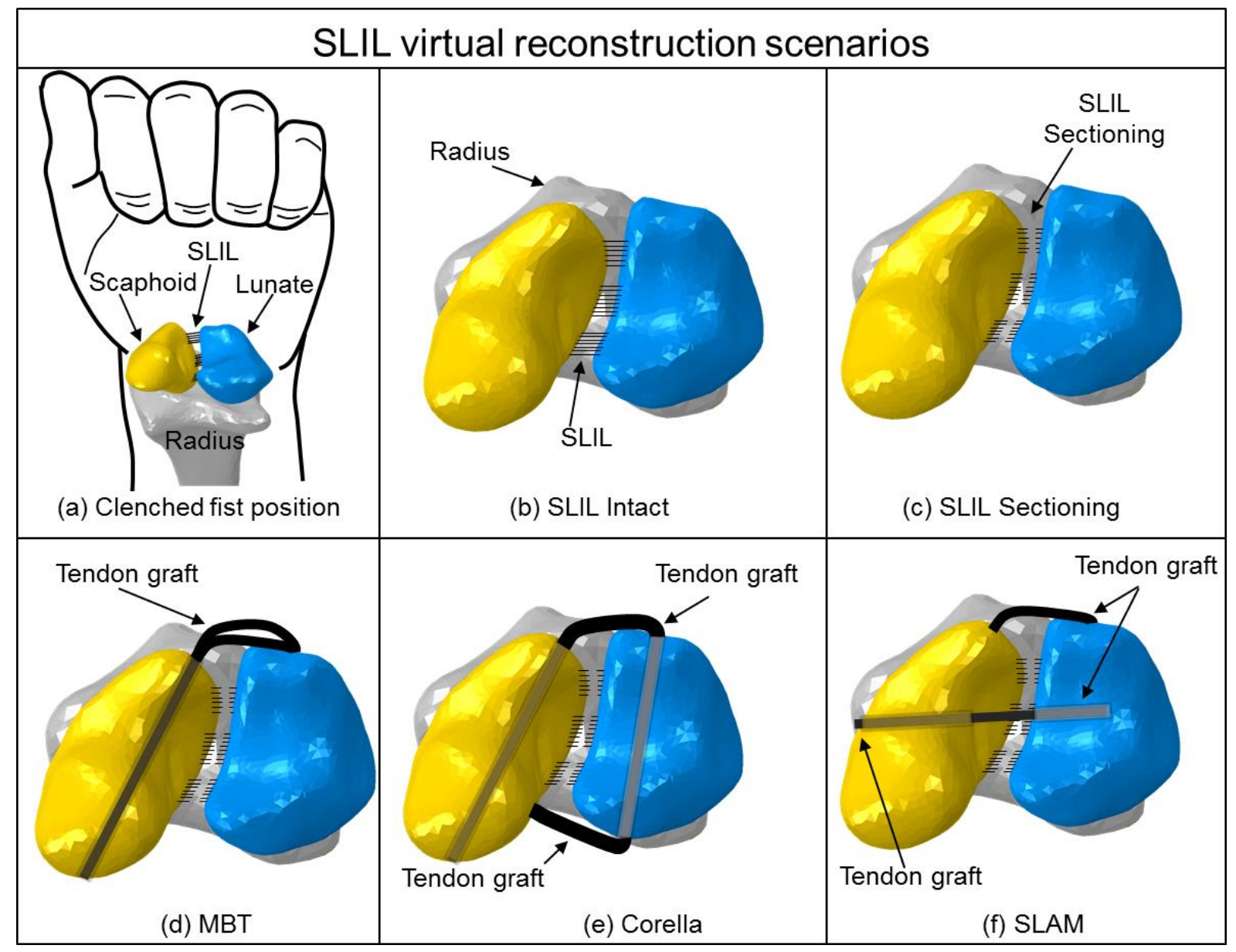

Figure 5 SLIL virtual reconstruction scenarios. (a) Hand showing the scaphoid, lunate and intact SLIL ligament in neutral clenched fist position. (b) SLIL intact ligament. (c) SLIL sectioning. (d) Modified Brunelli Tenodesis (MBT). (e) Corella (f) SLAM model. 


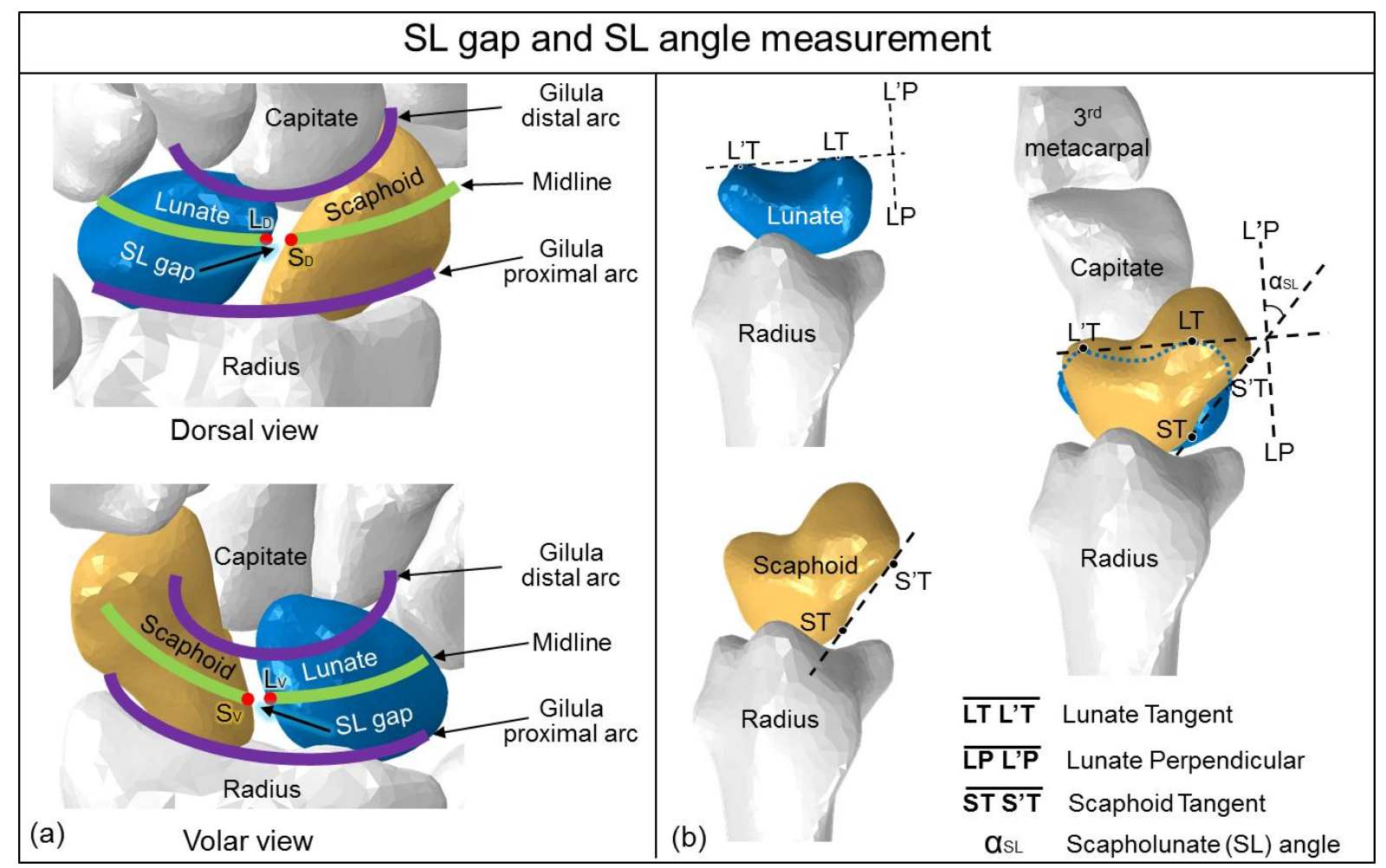

Figure 6 (a) The SL gap from FE model, calculated from the distance between two points defined at the intersection of the midline of the Gilula arcs (proximal and distal) and the border of the lunate and scaphoid (b) SL angle measured between two lines in the lateral view. Line 1(LP-L'P) perpendicular to the tangent line of the two distal poles of the lunate (LT-L'T). Line 2 (ST-S'T) a tangent line of the proximal and distal margin of the scaphoid at palmar side. The SL angle is the angle between Line 1 and Line 2 


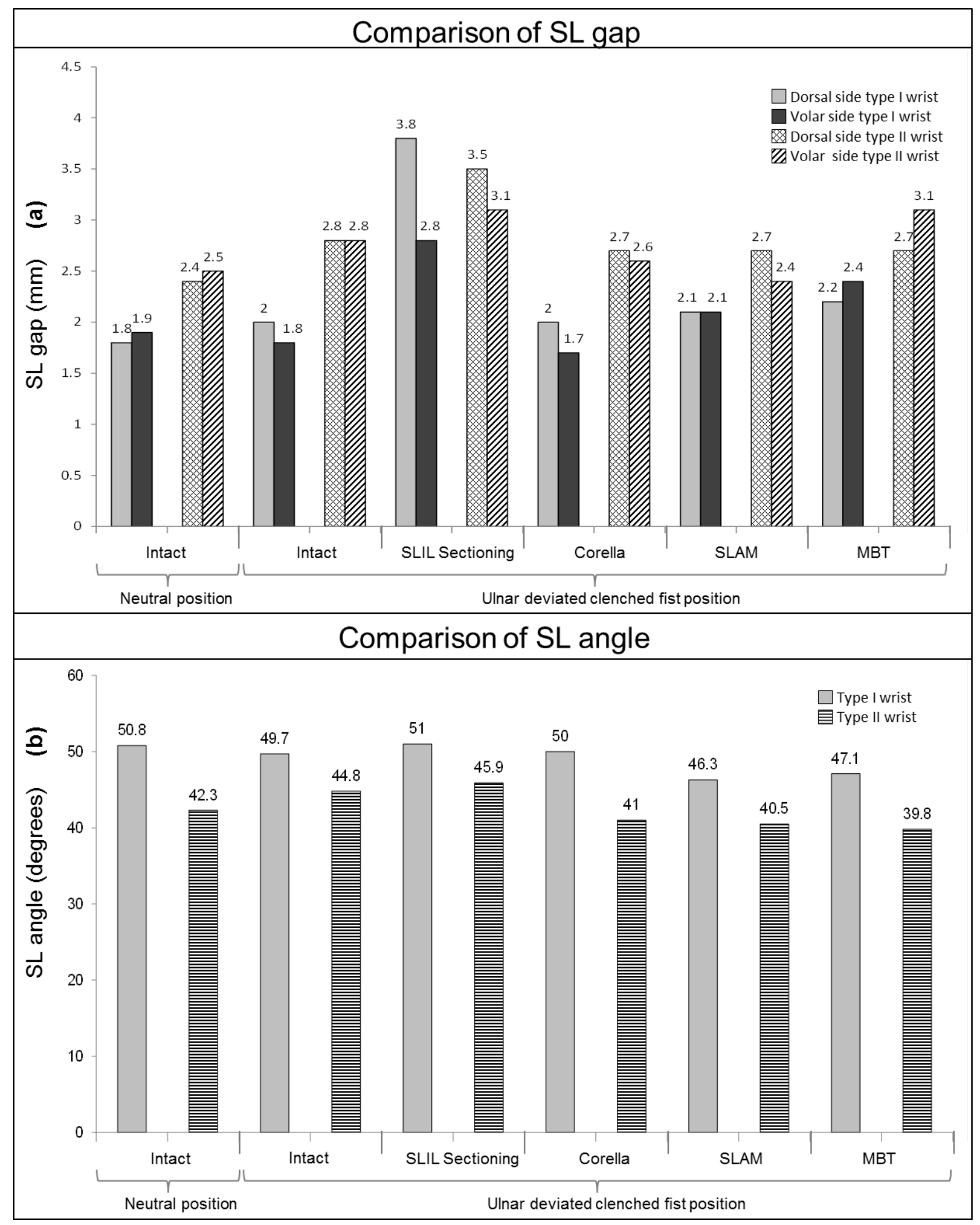

Figure 7 (a) Comparison of the predicted SL gap at dorsal and volar sides in the Finite Element models for the intact position, SLIL model and the 3 reconstruction techniques at the ulnar deviated clench fist position for the both types of human wrist. (b) Comparison of the predicted SL angle for the intact ligament in neutral position plus intact ligament, SLIL sectioning and the 3 reconstruction techniques with the model at the ulnar deviated clench fist position for the both types of wrist. 


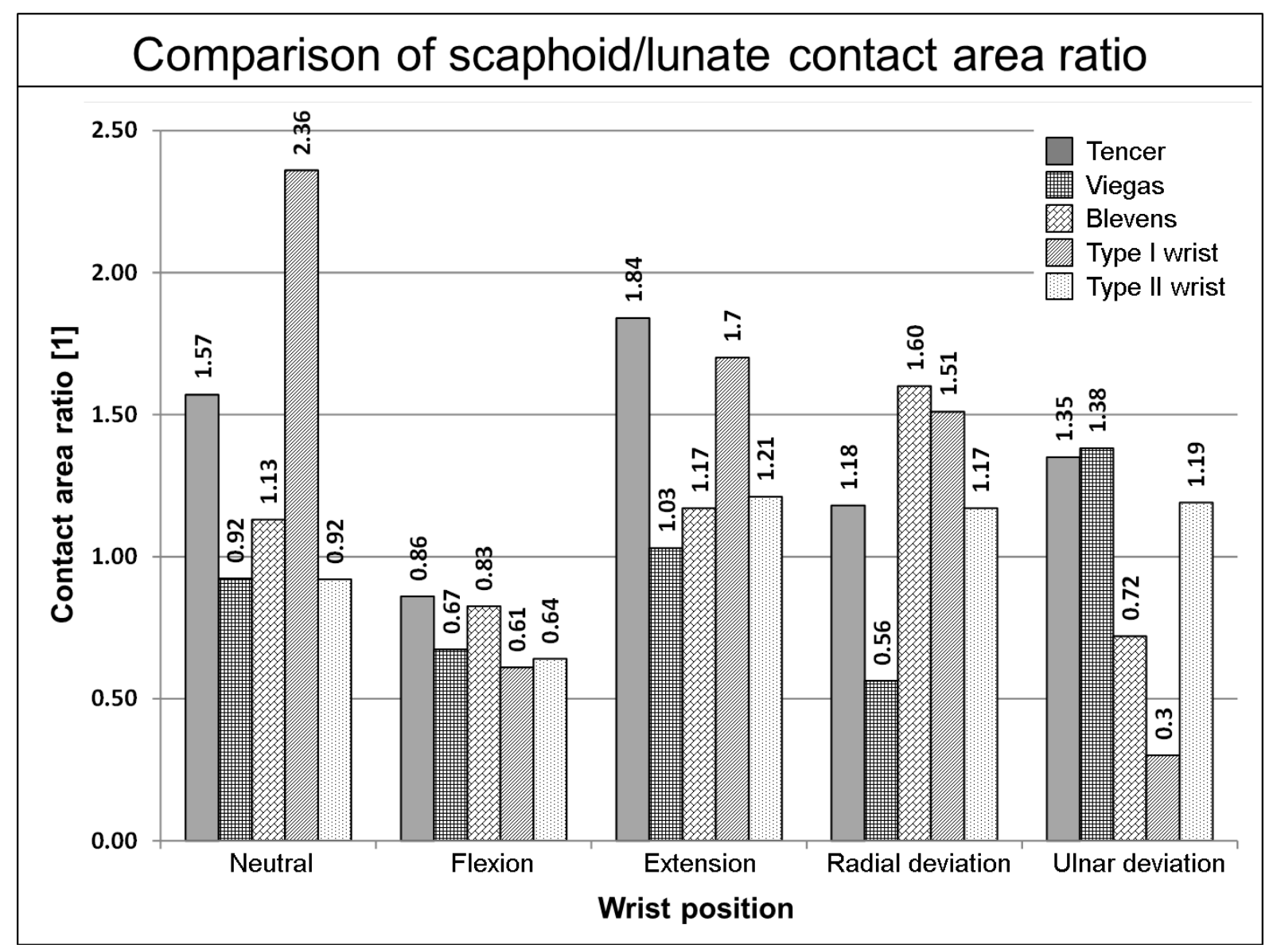

Figure 8 Comparison of the scaphoid/lunate contact area ratio between Finite Element model predictions and experimental values reported in the literature. 


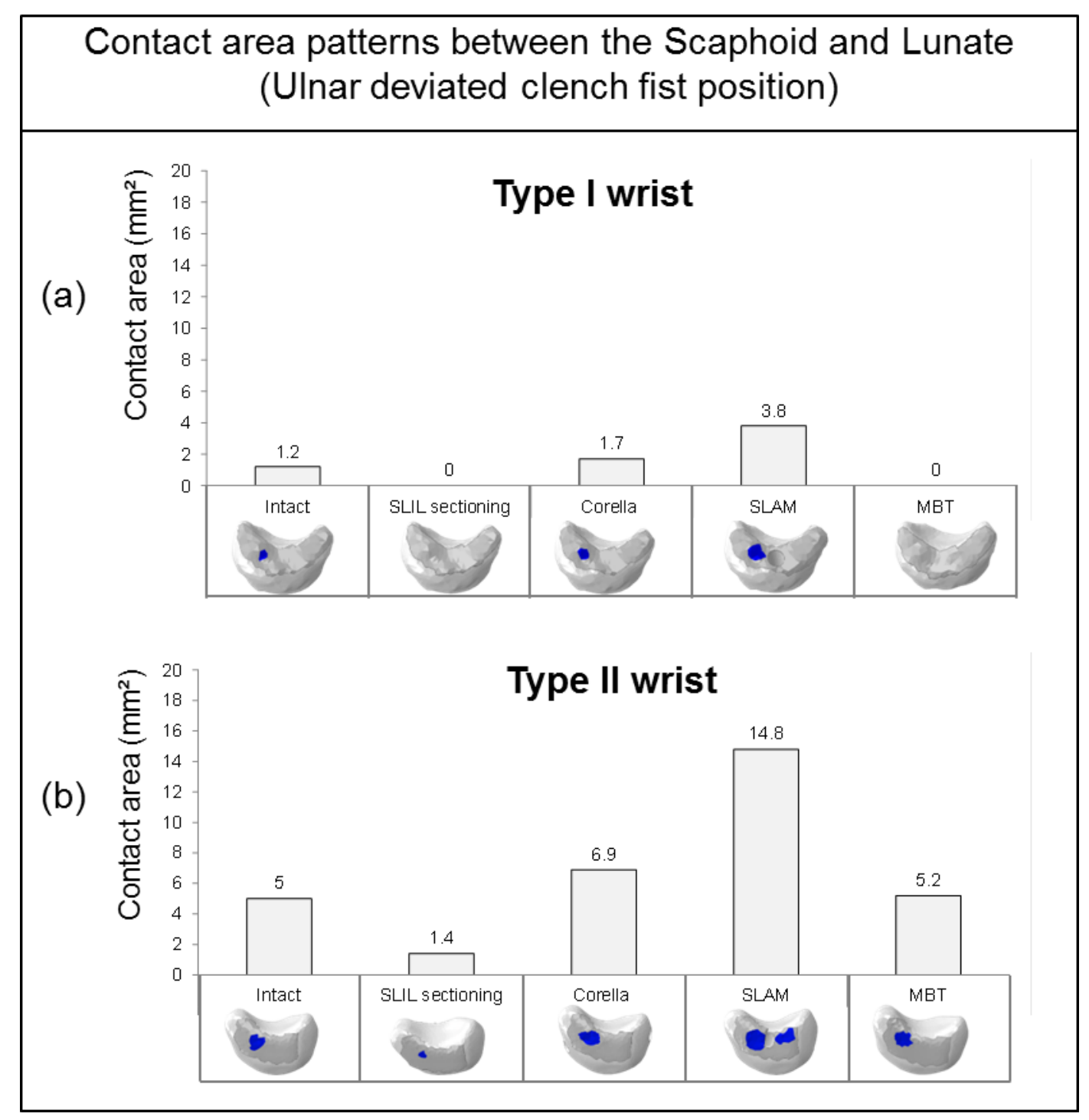

Figure 9 Comparison of the contact area patterns at the Scaphoid - Lunate interface for the intact, SLIL Sectioning and the 3 tenodesis techniques. 


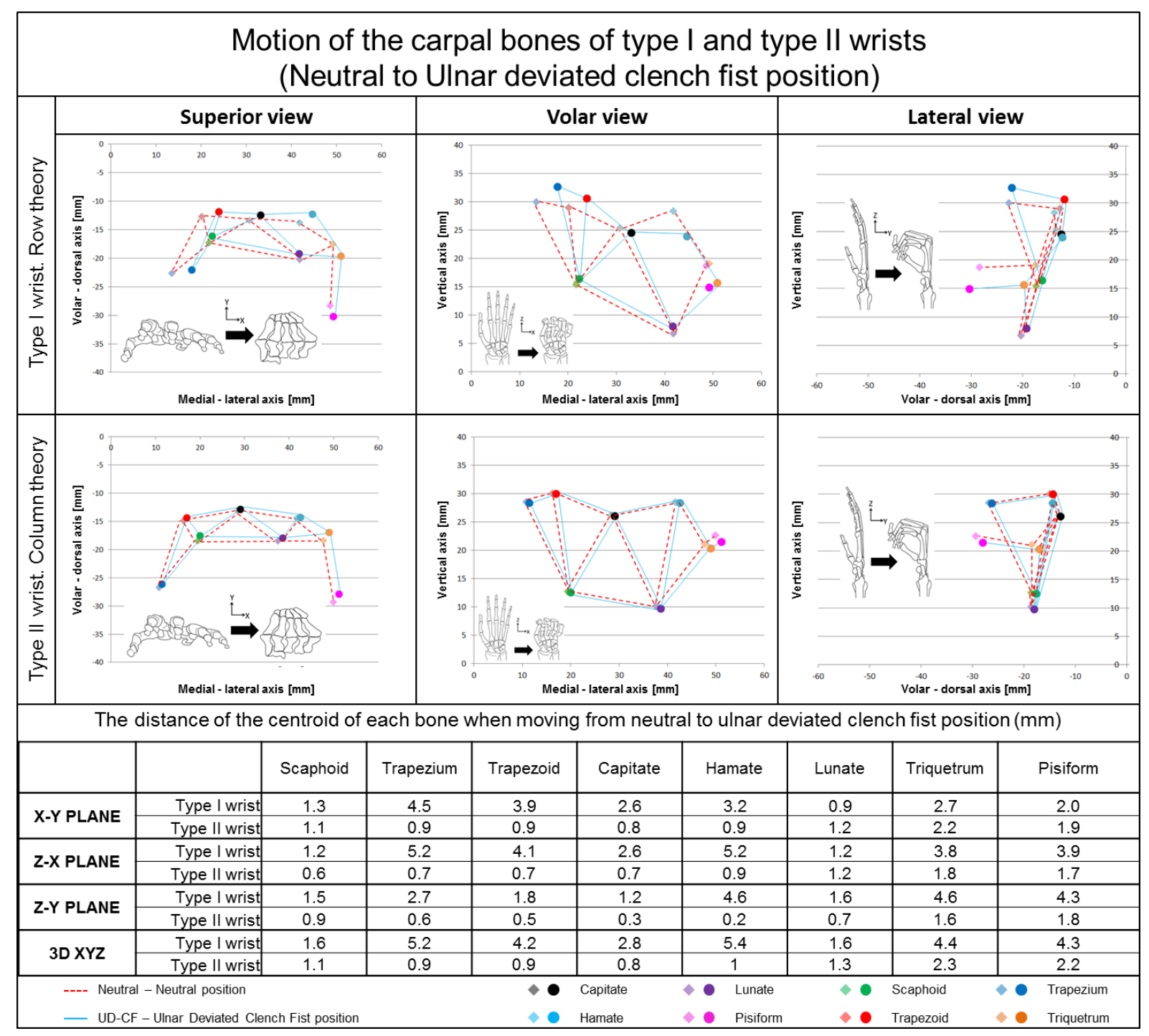

Figure 10 Comparison of the motion for type I and type II wrist during ulnar deviation. 


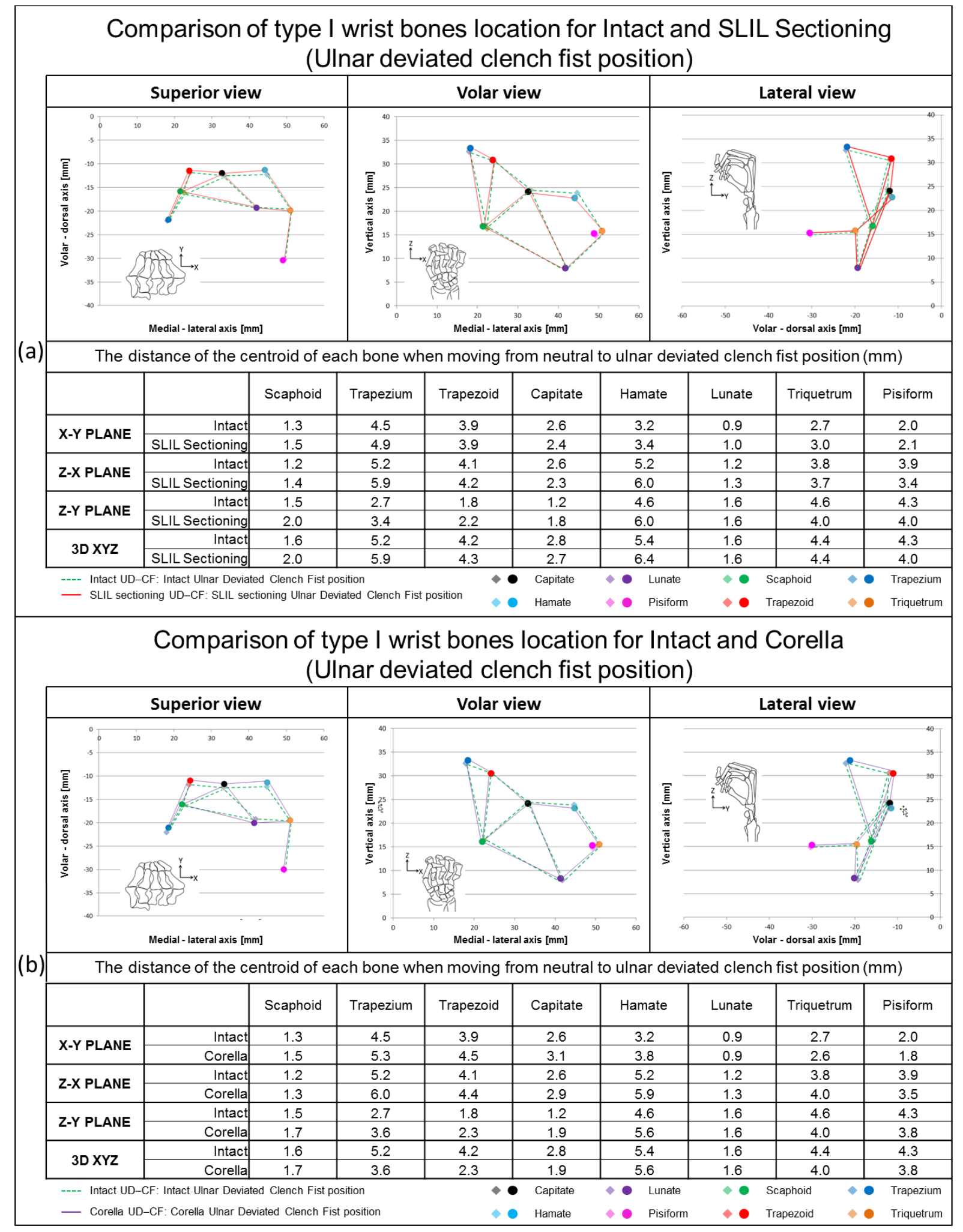

Figure 11 (a) Location of the bones after clench fist position comparing the intact scenario to the SLIL sectioning scenario for a type I wrist. (b) Location of the bones after clench fist position comparing the intact scenario to the Corella for a type I wrist. 


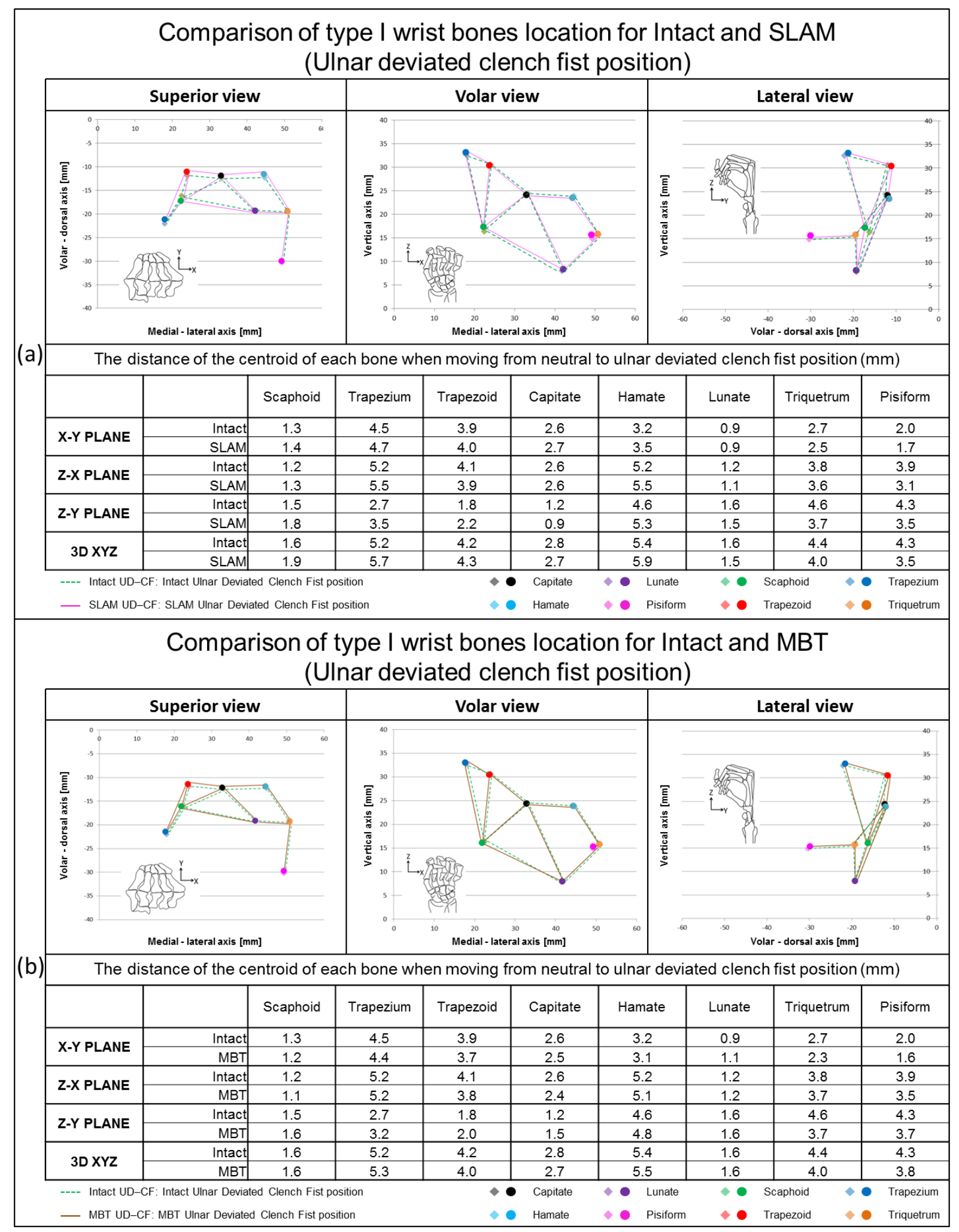

Figure 12 (a) Location of the bones after clench fist position comparing the intact scenario to the SLAM

technique for a type I wrist. (b) Location of the bones after clench fist position comparing the intact scenario to the MBT for a type I wrist. 


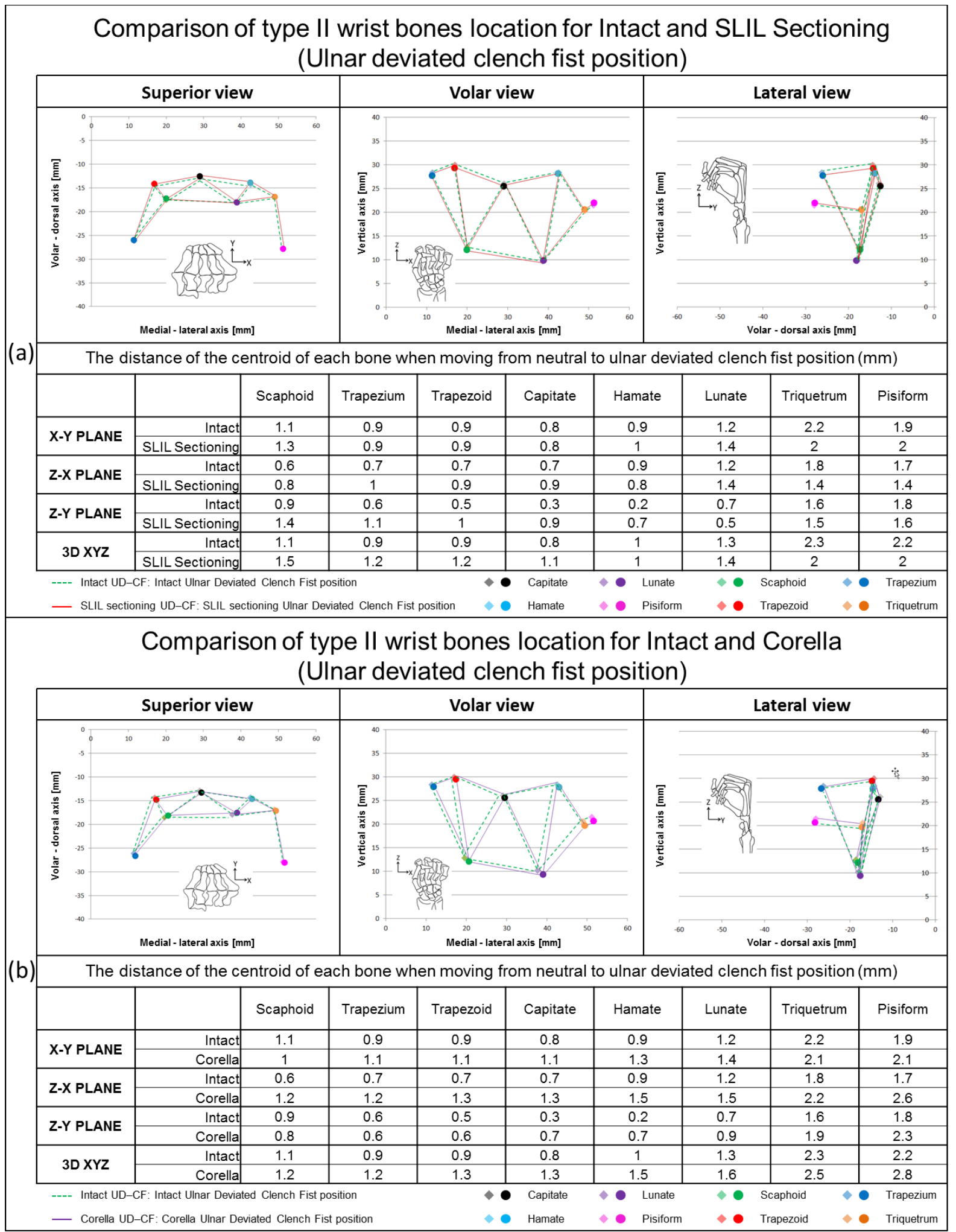

Figure 13 (a) Location of the bones after clench fist position comparing the intact scenario to the SLIL sectioning scenario for a type II wrist. (b) Location of the bones after clench fist position comparing the intact scenario to the Corella technique for a type II wrist. 


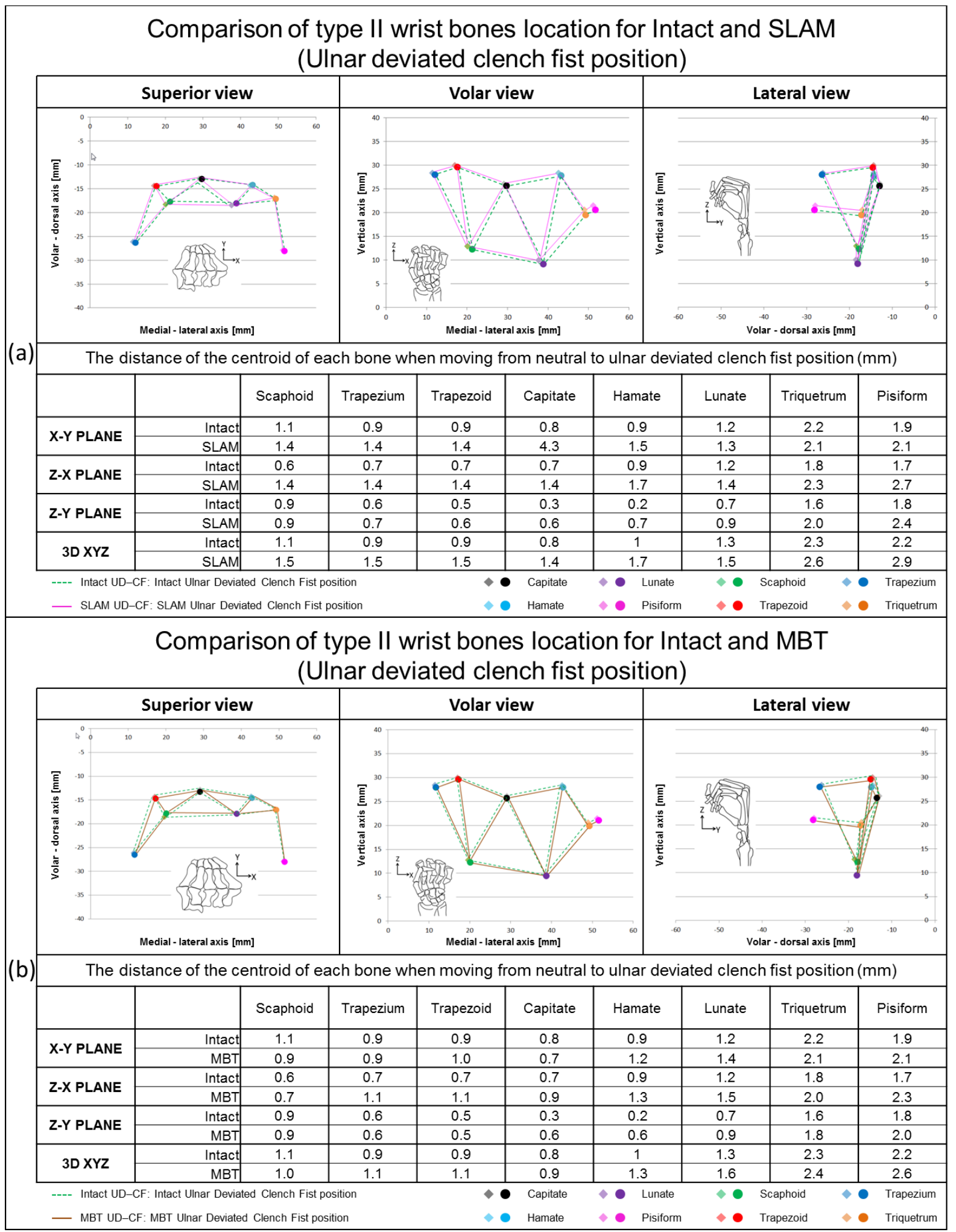

Figure 14 (a) Location of the bones after clench fist position comparing the intact scenario to the SLAM technique for a type II wrist. (b) Location of the bones after clench fist position comparing the intact scenario to the MBT for a type II wrist. 